\title{
Generalized Ornstein-Uhlenbeck Processes and Infinite Particle Branching Brownian Motions
}

\author{
By \\ Richard A. HOLLEY*(1)(2) \\ and \\ Daniel W. STROOCK*(1)
}

\section{Introduction}

The origins of this paper lie in a question posed to us by Frank Spitzer who, in fact, ended up solving most of his problem on his own. His problem is the following. Consider an infinite system of independent $d$-dimensional branching Brownian motions which at their branching times disappear or double with equal probabilities, and assume that the initial distribution of the system is a Poisson point process. Denote by $\eta_{t}(\Gamma), t \geq 0$ and $\Gamma \in \mathscr{B}_{{ }^{d}}$, the number of particles in $\Gamma$ at time $t$. The first question is to determine if $\eta_{t}(\cdot)$ has a non-trivial limiting distribution (as measure-valued random variables) when $t \rightarrow \infty$. The answer is yes if $d \geq 3$ and no if $d=1$ or 2 (cf. [2], [4], [6] and for a related situation [3]). The question in which Spitzer was interested is what happens if (when $d \geq 3$ ) one appropriately rescales the limiting random variable $\eta_{\infty}$. To be precise, given $\alpha>0$, define for bounded $\Gamma \in \mathscr{B}_{{ }^{d}}$ :

$$
\eta^{(\alpha)}(\Gamma)=\frac{\eta_{\infty}(\alpha \Gamma)-\alpha^{d}|\Gamma|}{\alpha^{(d+2) / 2}},
$$

where $|\Gamma|$ denotes the Lebesgue measure of $\Gamma$; and think of $\eta^{(\alpha)}$ as

Communicated by K. Itô, November 7, 1977.

* Department of Mathematics, University of Colorado, Boulder, Colorado 80302, U.S. A.

(1) Research partially supported by N.S. F. Grants MPS 74-18926 and MCS 77-14881.

(2) Alfred PIS.oan Fellow. 
taking values among tempered distributions. Using some very pretty moment calculations, Spitzer found that as $\alpha \rightarrow \infty$ the distribution of $\eta^{(a)}$ tends to the Gaussian measure on tempered distributions whose characteristic function is

$$
\exp \left[-1 / 2\left(\varphi,\left(-\Delta^{-1}\right) \varphi\right)\right]
$$

for test functions $\varphi$. Independently, Dawson [2] recently discovered the same result.

The idea in this paper is to study what happens if one reverses the limit procedures just described (a similar but somewhat different limit problem was studied by Martin-Lof in [7]. In fact a minor modification of our procedure can be used to prove his result). That is, consider

$$
\eta_{t}^{(\alpha)}(\Gamma)=\frac{\eta_{\alpha^{2} t}(\alpha \Gamma)-\alpha^{d}|\Gamma|}{\alpha^{(d+2) / 2}},
$$

and think of $\eta^{(a)}$ as a continuous process with values in tempered distributions. What we have shown is that, when $d \geq 3$, the distribution of $\eta^{(\alpha)}$ as $\alpha \rightarrow \infty$ tends to the distribution of the Ornstein-Uhlenbeck process $N$. determined by the relation:

$$
N_{t}-\int_{0}^{t} 1 / 2 \Delta N_{s} d s=\mathscr{W}_{t}
$$

where $\mathscr{W}$ is the standard Siegel process on tempered distributions. When $d=2$ the situation reduces to one which has been studied extensively by Dawson (see [2], [13], and [14] and the remark following Theorem (4.11) below.)

We next classify all the invariant measures for the process $N$. and show that they are all closely related to the Gaussian measure found by Spitzer et. al. Finally, we have isolated a dense $F_{\text {o }}$ subspace of the tempered distributions on which $N$. is concentrated and for which $N$. is ergodic.

Throughout this paper we will use $\mathscr{S}\left(R^{d}\right)$ to denote the Schwartz space of real-valued $C^{\infty}$-function which together with all their derivatives are rapidly decreasing. The space $\mathscr{S}^{\prime}\left(R^{d}\right)$ is then the space of real-valued tempered distributions. At only one point (cf. Lemma 
(5.17)) will we need complex valued tempered distributions, and we will then use the notation $\mathscr{S}_{c}\left(R^{d}\right)$. Also, $\|\cdot\|$ denotes $L^{2}$-norm throughout.

It should be obvious that we are deeply in debt to Frank Spitzer, and we take this opportunity to thank him.

\section{§1. Existence and Uniqueness of Generalized O.-U. Processes}

Throughout this section we will be using the following notation and assumptions. Let $A: \mathscr{S}\left(R^{d}\right) \rightarrow \mathscr{S}\left(R^{d}\right)$ be a bounded linear operator which admits a non-positive definite self-adjoint extention $\bar{A}$ on $L^{2}\left(R^{d}\right)$. We further assume that there is a strongly continuous semi-group $\left\{T_{t} ; t \geq 0\right\}$ of bounded linear operators on $\mathscr{S}\left(R^{d}\right)$ into itself such that

$$
N\left(T_{t} \varphi\right)-N(\varphi)=\int_{0}^{t} N\left(A T_{s} \varphi\right) d s, \quad t \geq 0
$$

for all $N \in \mathscr{S}^{\prime}\left(R^{d}\right)$ and $\varphi \in \mathscr{S}\left(R^{d}\right)$. It is easy to check that $T_{t} \varphi=e^{t \bar{A}} \varphi$ a. e. for $\varphi \in \mathscr{S}\left(R^{d}\right)$ (here $e^{t \bar{A}}$ denotes the semi-group of self-adjoint contractions on $L^{2}\left(R^{d}\right)$ generated by $\left.\bar{A}\right)$ and that $A T_{t} \varphi=T_{t} A \varphi$. We will of ten use the notation

$$
\varphi_{t}=T_{t} \varphi
$$

(1. 3) Lemma: If $t \rightarrow X_{t}$ is a continuous map from $[0, \infty)$ into $\mathscr{S}^{\prime}\left(R^{d}\right)$ and if $\varphi \in \mathscr{S}\left(R^{d}\right)$, then $(s, t) \rightarrow X_{s}\left(\varphi_{t}\right)$ is continuous on $([0, \infty))^{2}$.

Proof: Let $\left(s_{0}, t_{0}\right)$ be given. Since $\left\{X_{s}: s \in\left[0, s_{0}+1\right]\right\}$ is compact in $\mathscr{S}^{\prime}\left(R^{d}\right)$ and $t \rightarrow \varphi_{t}$ is strongly continuous,

$$
\lim _{t \rightarrow t_{0}} \sup _{s \in\left[0, s_{0}+1\right]}\left|X_{s}\left(\varphi_{t}\right)-X_{s}\left(\varphi_{t_{0}}\right)\right|=0
$$

Thus

$$
\varlimsup_{(s, t) \rightarrow\left(s_{0}, t_{0}\right)}\left|X_{s}\left(\varphi_{t}\right)-X_{s_{0}}\left(\varphi_{t_{0}}\right)\right| \leq \varlimsup_{s \rightarrow s_{0}}\left|X_{s}\left(\varphi_{t_{0}}\right)-X_{s}\left(\varphi_{t_{0}}\right)\right|=0 .
$$

Q. E. D. 
Let $\Omega=C\left([0, \infty), \mathscr{S}^{\prime}\left(R^{d}\right)\right)$. For $t \geq 0$, set $\mathscr{M}_{t}=\sigma\left(N_{s}(\varphi): 0 \leq s \leq t\right.$ and $\left.\varphi \in \mathscr{S}\left(R^{d}\right)\right)$ and put $\mathscr{M}=\sigma\left(\bigcup_{t \geq 0} \mathscr{M}_{t}\right)$. Finally, let $B: L^{2}\left(R^{d}\right) \rightarrow L^{2}\left(R^{d}\right)$ be a bounded linear operator. The next result is central to our analysis.

(1.4) Theorem: Let $P$ be a probability measure on $(\Omega, \mathscr{M})$ such that

$$
\begin{gathered}
f\left(N_{t \wedge \tau}(\varphi)\right)-\int_{0}^{t \wedge \tau} N_{u}(A \varphi) f^{\prime}\left(N_{u}(\varphi)\right) d u- \\
\frac{\|B \varphi\|^{2}}{2} \int_{0}^{t \wedge \tau} f^{\prime \prime}\left(N_{u}(\varphi)\right) d u
\end{gathered}
$$

is a martingale relative to $\left(\Omega, \mathscr{M}_{t}, P\right)$ for all $f \in C_{0}^{\infty}\left(R^{d}\right), \varphi \in \mathscr{S}\left(R^{d}\right)$ and stopping times $\tau$ such that $\sup _{\boldsymbol{\omega}} \sup _{t \geq 0}\left|N_{t \wedge \tau(\omega)}(A \varphi, \omega)\right|<\infty$. Then, for all $f \in C_{0}^{\infty}\left(R^{d}\right)$ and $\varphi \in \mathscr{S}\left(R^{d}\right)$,

$$
f\left(N_{t}(\varphi)-\int_{0}^{t} N_{u}(A \varphi) d u\right)-\frac{\|B \varphi\|^{2}}{2} \int_{0}^{t} f^{\prime \prime}\left(N_{u}(\varphi)-\int_{0}^{u} N_{s}(A \varphi) d s\right) d u
$$

is a martingale relative to $\left(\Omega, \mathscr{M}_{t}, P\right)$. Moreover, for all $0 \leq s<t$, $\varphi \in \mathscr{S}\left(R^{d}\right)$, and $\Gamma \in \mathscr{B}_{R^{d}}$ :

$$
\begin{aligned}
& P\left(N_{t}(\varphi) \in \Gamma \mid \mathscr{M}_{s}\right)= \\
& \left.\quad \int_{r} g\left(\int_{0}^{t-s} \|\left. B \varphi_{u}\right|^{2} d u, y-N_{s}\left(\varphi_{t-s}\right)\right) d y \quad \text { (a.s., } P\right),
\end{aligned}
$$

where $g(t, y)=\frac{1}{(2 \pi t)^{1 / 2}} e^{-y^{2} / 2 t}$. In particular, the condition (1.5) on $P$ plus a knowledge of $P \mid \mathscr{M}_{0}$ uniquely determines $P$ on $(\Omega, \mathscr{M})$. Finally, $P$ satisfies this condition if and only if the distribution of $N_{t}-T_{t} N_{0}$ also satisfies it.

Proof: Given $\varphi \in \mathscr{S}\left(R^{d}\right)$, define

$$
\tau_{n}=\left(\inf \left\{t \geq 0:\left|N_{t}(A \varphi)\right| \geq n\right\}\right) \wedge n .
$$

Then $\tau_{n} \uparrow \infty$ as $n \rightarrow \infty$ and

$$
f\left(N_{t \wedge \tau_{n}}(\varphi)\right)-\int_{0}^{t \wedge \tau_{n}} N_{u}(A \varphi) f^{\prime}\left(N_{u}(\varphi)\right) d u-\frac{\|B \varphi\|^{2}}{2} \int_{0}^{t \wedge \tau_{n}} f^{\prime \prime}\left(N_{u}(\varphi)\right) d u
$$

is a $P$-martingale for each $n$. From this fact it is easy to see that 


$$
\begin{aligned}
f\left(N_{t \wedge \tau_{n}}(\varphi)-\int_{0}^{t \wedge \tau}{ }_{n} N_{u}(A \varphi) d u\right)- \\
\frac{\|B \varphi\|^{2}}{2} \int_{0}^{t \wedge \tau_{n}} f^{\prime \prime}\left(N_{u}(\varphi)-\int_{0}^{u} N_{s}(A \varphi) d s\right) d u
\end{aligned}
$$

is a $P$-martingale for each $n$. But now we can let $n \rightarrow \infty$ and thereby conclude that

$$
f\left(N_{t}(\varphi)-\int_{0}^{t} N_{u}(A \varphi) d u\right)-\frac{\|B \varphi\|_{1}^{2}}{2} \int_{0}^{t} f^{\prime \prime}\left(N_{u}(\varphi)-\int_{0}^{u} N_{s}(A \varphi) d s\right) d u
$$

is a $P$-martingale. Using Theorem 2.1 in [12], we next see that for any $\varphi \in \mathscr{S}\left(R^{d}\right)$ and $s \geq 0$ :

$$
X_{\varphi}^{s}(t)=\exp \left[i\left(N_{t \vee s}(\varphi)-N_{s}(\varphi)-\int_{s}^{t \vee s} N_{u}(A \varphi) d u\right)+\frac{\|B \varphi\|^{2}}{2}(t \vee s-s)\right]
$$

is a $P$-martingale. It is our plan to prove from this that for all $T>0$ and $\varphi \in \mathscr{S}\left(R^{d}\right)$ :

$$
Y_{\varphi}^{U}(t)=\exp \left[i N_{t \wedge T}\left(\varphi_{T-t \wedge T}\right)+\left.\frac{1}{2} \int_{0}^{t \wedge T}|| B \varphi_{T-u}\right|^{2} d u\right]
$$

is a $P$-martingale. To this end, let $0 \leq t_{1}<t_{2} \leq T$ be given. Then, by the continuity of $(s, t) \rightarrow N_{s}\left(\varphi_{t}\right)$, it is easy to show that

$$
\frac{Y_{\varphi}^{T}\left(t_{2}\right)}{Y_{\varphi}^{T}\left(t_{1}\right)}=\lim _{n \rightarrow \infty} \prod_{k=0}^{n-1} X_{\varphi_{T-\sigma_{n, k}}^{\sigma}}^{\sigma_{n, k}}\left(\sigma_{n, k+1}\right)
$$

where $\sigma_{n, k}=t_{1}+\frac{k}{n}-\left(t_{2}-t_{1}\right)$. Moreover, since that convergence is bounded, it takes place in $L^{1}(P)$. Thus, if $H \in \mathscr{M}_{t_{1}}$, then

$$
E^{P}\left[\frac{Y_{\varphi}^{T}\left(t_{2}\right)}{Y_{\varphi}^{T}\left(t_{1}\right)}, H\right]=\lim _{n \rightarrow \infty} E^{P}\left[\prod_{0}^{n-1} X_{\varphi_{T-\sigma_{n, k}}^{\sigma}}^{\sigma_{n, k}}\left(\sigma_{n, k+1}\right), H\right]
$$

But if $1 \leq m \leq n-1$, then

$$
E^{P}\left[\prod_{0}^{m-1} X_{\varphi_{T-\sigma_{n, k}}^{\sigma_{n, k}}}\left(\sigma_{n, k+1}\right), H\right]=E^{P}\left[\prod_{0}^{m-2} X_{\varphi_{T-\sigma_{n, k}}^{\sigma}}^{n_{n, k}}\left(\sigma_{n, k+1}\right), H\right],
$$

where it is understood that

$$
\prod_{0}^{-1} X_{\varphi_{T-\sigma_{n, k}}^{\sigma}}^{\sigma_{n, k}}\left(\sigma_{n, k+1}\right) \equiv 1 .
$$

It follows that 


$$
E^{P}\left[Y_{\varphi}^{T}\left(t_{2}\right) / Y_{\varphi}^{T}\left(t_{1}\right), H\right]=P(H), H \in \mathscr{M}_{t_{1}},
$$

which is to say that $\left(Y_{\varphi}^{T}(t), \mathscr{M}_{t}, P\right)$ is a martingale. It particular, if $0 \leq s<t$, then

$$
\begin{aligned}
E^{P}\left[e^{i N(\varphi)} \mid \mathscr{M}_{s}\right] & =e^{-1 / 2 \int_{0}^{t} t_{B \varphi_{u}} \|^{2} d u} E^{P}\left[Y_{\varphi}^{t}(t) \mid \mathscr{M}_{s}\right] \\
& =e^{-1 / 2 \int_{0}^{t}\left\|B \varphi_{s}\right\|^{2} d u} Y_{\varphi}^{t}(s) \\
& \left.=e^{i N_{s}\left(\varphi_{t-s}\right)+1 / 2 \int_{0}^{t-s_{\| B \varphi_{u}} \|^{2} d u}} \quad \text { (a. s., } P\right),
\end{aligned}
$$

and clearly (1.5) is an easy consequence of this.

The assertion about the uniqueness of $P$ is obtained by standard Markovian reasoning from (1.6). Finally, the statement about translating $N_{t}$ by $T_{t} N_{0}$ is an easy consequence of standard manipulations with martingales.

Q. E. D.

(1.7) Corollary: Let $P$ be as in Theorem 1.4 and assume in addition that $P\left(N_{0}=0\right)=1$. Then for each $T>0$ and $\varphi \in \mathscr{S}\left(R^{d}\right)$ :

$$
E^{P}\left[\sup _{0<t<T}\left|N_{t}(\varphi)\right|^{2}\right] \leq 8(1+T)^{3} \mid i B \|^{2}\left(\|\varphi\|^{2}+\|A \varphi\|^{2}\right) .
$$

In particular,

$$
f\left(N_{t}(\varphi)\right)-\int_{0}^{t} N_{u}(A \varphi) f^{\prime}\left(N_{u}(\varphi)\right) d u-\frac{\|B \varphi\|^{2}}{2} \int_{0}^{t} f^{\prime \prime}\left(N_{u}(\varphi)\right) d u
$$

is a P-martingale for all $f \in C_{b}^{2}\left(R^{d}\right)$ and $\varphi \in \mathscr{S}\left(R^{d}\right)$.

Proof: The second part follows from the first part lapplied to $A \varphi$. To prove (1.8), note that by Theorem 1.4,

$$
f\left(N_{t}(\varphi)-\int_{0}^{t} N_{u}(A \varphi) d u\right)-\frac{\|B \varphi\|^{2}}{2} \int_{0}^{t} f^{\prime \prime}\left(N_{u}(\varphi)-\int_{0}^{u} N_{s}(A \varphi) d s\right) d u
$$

is a $P$-martingale for all $f \in C_{0}^{\infty}\left(R^{d}\right)$. From this fact it is not hard to verify that the same is true for $f \in C^{2}(R)$ which, together with their first two derivatives, grow no faster than $C_{1} e^{C_{2}|x|}$ for some finite $C_{1}$ and $C_{2}$. Thus

$$
N_{t}(\varphi)-\int_{0}^{t} N_{u}(A \varphi) d u
$$

and 


$$
\left(N_{t}(\varphi)-\int_{0}^{t} N_{u}(A \varphi) d u\right)^{2}-\|B \varphi\|^{2} t
$$

are $P$-martingales. By Doob's inequality, it follows that

$$
\begin{gathered}
E^{P}\left[\sup _{0 \leq t \leq T}\left(N_{t}(\varphi)-\int_{0}^{t} N_{u}(A \varphi) d u\right)^{2}\right] \\
\leq 4 E^{P}\left[\left(N_{t}(\varphi)-\int_{0}^{T} N_{u}(A \varphi) d u\right)^{2}\right] \\
=4\|B \varphi\|^{2} T .
\end{gathered}
$$

Thus

$$
\begin{gathered}
E^{P}\left[\sup _{0 \leq t \leq T}\left|N_{t}(\varphi)\right|^{2}\right] \leq 2 E^{P}\left[\left(\int_{0}^{T}\left|N_{u}(A \varphi)\right| d u\right)^{2}\right]+8\|B \varphi\|^{2} T \\
\leq T\left(2 E^{P}\left[\int_{0}^{T}\left|N_{u}(A \varphi)\right|^{2} d u\right]+8\|B \varphi\|^{2}\right) .
\end{gathered}
$$

By (1.6)

$$
\begin{aligned}
E^{P}\left[\int_{0}^{T}\left|N_{u}(\varphi)\right|^{2} d u\right] & =\int_{0}^{T} d u \int_{0}^{u}|| B A \varphi_{v} \|^{2} d v \\
& \leq \frac{\|B\|^{2} T^{2}}{2} \|\left. A \varphi\right|^{2} .
\end{aligned}
$$

Hence

$$
\begin{aligned}
E^{P}\left[\sup _{0 \leq t \leq T}\left|N_{t}(\varphi)\right|^{2}\right] & \leq T\|B\|^{2}\left(T^{2}\|A \varphi\|^{2}+8\|||\|^{2}\right) \\
& \leq 8(1+T)^{3} \|\left. B_{i}\right|^{2}\left(\left\|\left.A \varphi\right|^{2}+\right\| \varphi \|^{2}\right) .
\end{aligned}
$$

Q. E. D.

From now on we will say that $P_{M}$ on $(\Omega, \mathscr{M})$ is the OrnsteinUhlenbeck process with characteristics $A$ and $B$ starting from $M \in \mathscr{S}^{\prime}\left(R^{d}\right)$ if $P_{M}\left(N_{0}=M\right)=1$ and $P_{M}$ satisfies the conditions of Theorem 1.4. By that theorem, we know that there is for each $M$ at most one such $P_{M}$ and $P_{M}$ is related to $P_{0}$ by the fact that $N_{t}-T_{t} M$ has distribution under $P_{M}$ equal to $P_{0}$. Thus, if $P_{0}$ exists, then $P_{M}$ exists for each $M \in \mathscr{S}^{\prime}\left(R^{d}\right)$ and the family $\left\{P_{M}: M \in \mathscr{S}^{\prime}\left(R^{d}\right)\right\}$ forms a strong Markov Feller process. (The assertion about the Markov property is an easy consequence of uniqueness or can be derived directly from (1.6).) We now want to show that $P_{0}$ always exists.

For each multi-index $\alpha=\left(\alpha_{1}, \ldots, \alpha_{d}\right), \alpha_{i} \geq 0$ for $1 \leq j \leq d$, let 
$h_{\alpha}(x)=h_{\alpha_{1}}\left(x_{1}\right) \ldots h_{\alpha_{d}}\left(x_{d}\right)$ where $h_{k}$ denotes the $k^{t h}$ Hermite function (cf. A. 7). Given $n \geq 0$, define $\Pi_{n}: L^{2}\left(R^{d}\right) \rightarrow \mathscr{S}\left(R^{d}\right)$ by

$$
\Pi_{n} \varphi=\sum_{|\alpha| \leq n}\left(\varphi, h_{\alpha}\right) h_{\alpha}
$$

It is well-known that $\Pi_{n} \varphi \rightarrow \varphi$ as $n \rightarrow \infty$ in $L^{2}\left(R^{d}\right)$ for all $\varphi \in L^{2}\left(R^{d}\right)$ and that $\Pi_{n} \varphi \rightarrow \varphi$ in $\mathscr{S}\left(R^{d}\right)$ for all $\varphi \in \mathscr{S}\left(R^{d}\right)$ (cf. A. 15).

In order to carry out our construction we proceed as follows. For $n \geq 0$, set $D_{n}=\operatorname{card}\{\alpha:|\alpha| \leq n\}$ and define

$$
a_{\alpha, \beta}=\sum_{|r| \leq n}\left(B h_{\alpha}, h_{\gamma}\right)\left(B h_{\beta}, h_{r}\right)
$$

and

$$
b_{\alpha, \beta}=\left(A h_{\alpha}, h_{\beta}\right)
$$

for $|\alpha|,|\beta| \leq n$. Next let

$$
L^{(n)}=\frac{1}{2} \sum_{|\alpha|,|\beta| \leq n} a_{\alpha, \beta} \frac{\partial^{2}}{\partial y_{\alpha} \partial y_{\beta}}+\sum_{|\alpha| \leq n}\left(\sum_{|\beta| \leq n} b_{\alpha, \beta} y_{\beta}\right) \frac{\partial}{\partial y_{\alpha}} .
$$

It is easily shown that there exists on $C\left([0, \infty), R^{D_{n}}\right)$ exactly one probability measure $Q^{(n)}$ such that $Q^{(n)}(y(0)=0)=1, E^{Q^{(n)}}\left[\sup _{0 \leq t \leq T}|y(t)|^{2}\right]$ $<\infty$ for all $T>0$, and $f(y(t))-\int_{0}^{t} L^{(n)} f(y(s)) d s$ is a $Q^{(n)}$-martingale for each $f \in C_{b}^{2}\left(R^{D_{n}}\right)$. (One can use, for example, Itô's stochastic differential equations as described in H. P. Mckean's [8].) Next define $t \rightarrow \tilde{N}_{t} \in \mathscr{S}^{\prime}\left(R^{d}\right)$ by

$$
\tilde{N}_{t}(\varphi)=\sum_{|\alpha| \leq n}\left(\varphi, h_{\alpha}\right) y_{\alpha}(t), \quad t \geq 0 \text { and } \varphi \in \mathscr{S}\left(R^{d}\right) .
$$

Given a $\varphi \in \mathscr{S}\left(R^{d}\right)$ and $f \in C_{0}^{\infty}\left(R^{1}\right)$, define $F \in C^{2}\left(R^{D_{n}}\right)$ by

$$
F(y)=f\left(\sum_{|\alpha| \leq n}\left(\varphi, h_{\alpha}\right) y_{\alpha}\right)
$$

Note that

$$
\begin{aligned}
L^{(n)} F(y) & =\frac{1}{2}\left(\sum_{|\alpha|,|\beta| \leq n} a_{\alpha, \beta}\left(\varphi, h_{\alpha}\right)\left(\varphi, h_{\beta}\right)\right) f^{\prime \prime}\left(\sum_{|\alpha| \leq n}\left(\varphi, h_{\alpha}\right) y_{\alpha}\right) \\
& +\left(\sum_{|\alpha| \leq n}\left(\varphi, h_{\alpha}\right)\left(\sum_{|\beta| \leq n} b_{\alpha, \beta} y_{\beta}\right)\right) f^{\prime}\left(\sum_{|\alpha| \leq n}\left(\varphi, h_{\alpha}\right) y_{\alpha}\right)
\end{aligned}
$$

and 


$$
\begin{aligned}
\sum_{|\alpha|,|\beta| \leq n} a_{\alpha, \beta}\left(\varphi, h_{\alpha}\right)\left(\varphi, h_{\beta}\right) & =\sum_{|\alpha|,|\beta| \leq n} \sum_{|\gamma| \leq n}\left(B h_{\alpha}, h_{\gamma}\right)\left(B h_{\beta}, h_{\gamma}\right)\left(\varphi, h_{\alpha}\right)\left(\varphi, h_{\beta}\right) \\
& =\sum_{|\gamma| \leq n}\left(B \Pi_{n} \varphi, h_{\gamma}\right)^{2}=\left.|| \Pi_{n} B \Pi_{n} \varphi\right|^{2} .
\end{aligned}
$$

Thus

$$
\begin{aligned}
L^{(n)} F(y(t)) & =\frac{1}{2}\left\|\Pi_{n} B \Pi_{n} \varphi\right\|^{2} f^{\prime \prime}\left(\tilde{N}_{t},(\varphi)\right) \\
& +\sum_{|a| \leq n}\left(\varphi, h_{\alpha}\right)\left(\sum_{|\beta| \leq n} b_{\alpha, \beta} y_{\beta}(t)\right) f^{\prime}\left(\tilde{N}_{t}(\varphi)\right) .
\end{aligned}
$$

But

$$
\begin{aligned}
\sum_{|\alpha| \leq n}\left(\varphi, h_{\alpha}\right) \sum_{|\beta| \leq n} b_{\alpha, \beta} y_{\beta}(t) & =\sum_{|\beta| \leq n}\left(\sum_{|\alpha| \leq n}\left(A h_{\alpha}, h_{\beta}\right)\left(\varphi, h_{\alpha}\right)\right) \tilde{N}_{t}\left(h_{\beta}\right) \\
& =\sum_{|\gamma| \leq n}\left(A \Pi_{n} \varphi, h_{\beta}\right) \tilde{N}_{t}\left(h_{\beta}\right)=\tilde{N}_{t}\left(\Pi_{n} A \Pi_{n} \varphi\right) .
\end{aligned}
$$

From these calculations, we see that if $P^{(n)}$ on $(\Omega, \mathscr{M})$ is the distribution of $\tilde{N}$. under $Q^{(n)}$, then $P^{(n)}\left(N_{0}=0\right)=1$ and for all $f \in C_{b}^{2}\left(R^{1}\right)$ and $\varphi \in \mathscr{S}\left(R^{d}\right)$ :

$$
f\left(N_{t}(\varphi)\right)-\int_{0}^{t} N_{s}\left(A_{n} \varphi\right) f^{\prime}\left(N_{s}(\varphi)\right) d s-\frac{\|\left. B_{n} \varphi_{!}^{!}\right|^{2}}{2} \int_{0}^{t} f^{\prime \prime}\left(N_{s}(\varphi)\right) d s
$$

is a $P^{(n)}$-martingale, where

$$
A_{n}=\Pi_{n} A \Pi_{n} \text { and } B_{n}=\Pi_{n} B \Pi_{n} .
$$

The next step is to show that $\left\{P^{(n)}: n \geq 0\right\}$ is weakly precompact. To this end, note that because $A$ is bounded from $\mathscr{S}\left(R^{d}\right)$ into itself, there is an $m_{0} \geq 0$ such that

$$
\|A \varphi\| \leq C \mid\|\varphi\|^{\left(m_{0}\right)}, \quad \varphi \in \mathscr{S}\left(R^{d}\right),
$$

for some $C<\infty$ (cf. A. 18). For this $m_{0}$, set $n_{0}=m_{0}+d+1$ and define $\|\cdot\|$ on $\mathscr{S}^{\prime}\left(R^{d}\right)$ by

$$
\|\left.|N|\right|_{1} ^{2}=\sum(2|\alpha|+d)^{-n_{0}}\left|N\left(h_{\alpha}\right)\right|^{2} .
$$

It is easy to check that

$$
\mathscr{H}_{n_{0}}=\left\{N \in \mathscr{S}^{\prime}\left(R^{d}\right):|| N|| \mid<\infty\right\}
$$

is a complete separable Hilbert space under $\|\cdot\| \|$ and that the dual of $\mathscr{H}_{n_{0}}$ can be identified in a natural way with the completion of $\mathscr{S}\left(R^{d}\right)$ under \|\|$\cdot \|^{\left(n_{0}\right)}$. Certainly it is enough for us to show that 
$P^{(n)}$ is concentrated on $C\left([0, \infty), \mathscr{H}_{n_{0}}\right)$ (which is an $F_{o}$-subset of $\left.C\left((0, \infty), \mathscr{S}^{\prime}\left(R^{d}\right)\right)\right)$ for all $n \geq 0$ and that $\left\{P^{(n)}: n \geq 0\right\}$ is weakly precompact subset of probability measures on $C\left([0, \infty), \mathscr{H}_{n_{0}}\right)$. Since under $P^{(n)}$ each $N .(\varphi)$ is continuous and $N .\left(h_{\alpha}\right) \equiv 0$ a. s. for $|\alpha|>n$, it is clear that $P^{(n)}$ is concentrated on $C\left([0, \infty), \mathscr{H}_{n_{0}}\right)$. Furthermore, by (1.6)

$$
\begin{aligned}
E^{P(n)} & {\left[\left|N_{t_{2}}(\varphi)-N_{t_{1}}(\varphi)\right|^{4}\right] } \\
& =3\left(\int_{0}^{t_{2}-t_{1}}\left\|B_{n} \varphi_{u}\right\|^{2} d u\right)^{2} \leq 3\left\|\left.B\right|^{2}\right\| \varphi \|^{2}\left(t_{2}-t_{1}\right)^{2}
\end{aligned}
$$

for $n \geq 0,0 \leq t_{1}<t_{2}$, and $\varphi \in \mathscr{S}\left(R^{d}\right)$. Thus, for any $m \geq 0, T>0$, and $\varepsilon>0$ :

$$
\lim _{\delta \downarrow 0} \sup _{n} P^{(n)}\left(\sup _{\substack{0 \leq t_{1}<t_{2} \leq T \\ t_{2}-t_{1}<\delta}} \max _{|\alpha| \leq m}\left|N_{t_{2}}\left(h_{\alpha}\right)-N_{t_{1}}\left(h_{\alpha}\right)\right| \geq \varepsilon\right)=0 .
$$

Since $P^{(n)}\left(N_{0}=0\right)=1$ for all $n \geq 0$, it only remains to check that for $T>0$ and $\varepsilon>0$ :

$$
\lim _{m \rightarrow \infty} \sup _{n} P^{(n)}\left(\sup _{0 \leq t \leq T}|i| \Pi_{m}^{\perp} N_{t}|| \geq \varepsilon\right)=0
$$

where $\Pi_{m}^{\perp} N$ is defined so that

$$
\Pi_{m}^{\perp} N(\varphi)=\sum_{|\alpha|>m}\left(\varphi, h_{\alpha}\right) N\left(h_{\alpha}\right), \quad \varphi \in \mathscr{S}\left(R^{d}\right) .
$$

But by (1.8) plus (1.15) :

$$
\begin{aligned}
E^{p^{(n)}} & {\left[\sup _{0 \leq t \leq T}\left|N_{t}\left(h_{\alpha}\right)\right|^{2}\right] } \\
& \leq 8(1+T)^{3}\|B\|^{2}\left(1+|| A_{n} h_{\alpha} \|^{2}\right) \\
& \leq 8 C(1+T)^{3}\|B\|^{2}\left(1+\left\||| h_{\alpha}\right\| \|^{\left(m_{0}\right)}\right) \\
& \leq 8 C(1+T)^{3}\|B\|^{2}(2|\alpha|+d+1)^{m_{0}}
\end{aligned}
$$

and so

$$
\begin{aligned}
& E^{P^{(n)}}\left[\left.\sup _{0 \leq t \leq T}\left|\| \Pi_{m}^{\perp} N_{t}\right|\right|^{2}\right] \\
& \quad \leq \sum_{|\alpha|>m}(2|\alpha|+d)^{-n_{0}} E^{P^{(n)}}\left[\sup _{0 \leq t \leq T}\left|N_{t}\left(h_{\alpha}\right)\right|^{2}\right] \\
& \quad \leq 8 C(1+T)^{3}|| B \|^{2} \sum_{|\alpha|>m} \frac{(2|\alpha|+d+1)^{m_{0}}}{(2|\alpha|+d)^{m_{0}+d+1}} .
\end{aligned}
$$


This proves that

$$
\lim _{m \rightarrow \infty} \sup _{n} E^{p^{(n)}}\left[\sup _{0 \leq t \leq T}\|\| \Pi_{m}^{\perp} N_{t} \|\left.\right|^{2}\right]=0
$$

which certainly implies (1.18).

The final step in the construction is to show that if $P$ is the limit of a convergent subsequence $\left\{P^{\left(n^{\prime}\right)}\right\}$ of $\left\{P^{(n)}: n \geq 0\right\}$, then $P\left(N_{0}=0\right)=1$, $E^{P}\left[\sup _{0 \leq t \leq T}\left|N_{t}(\varphi)\right|\right]<\infty$ for all $T>0$ and $\varphi \in \mathscr{S}\left(R^{d}\right)$, and

$$
f\left(N_{t}(\varphi)\right)-\int_{0}^{t} N_{u}(A \varphi) f^{\prime}\left(N_{u}(\varphi)\right) d u-\frac{\|B \varphi\|^{2}}{2} \int_{0}^{t} f^{\prime \prime}\left(N_{u}(\varphi)\right) d u
$$

is a $P$-martingale for each $f \in C_{0}^{\infty}\left(R^{d}\right)$ and $\varphi \in \mathscr{S}\left(R^{d}\right)$. But clearly $P\left(N_{0}=0\right) \geq \varlimsup_{n} P^{\left(n^{\prime}\right)}\left(N_{0}=0\right)=1$ and, by $(1.8)$,

$$
E^{P}\left[\sup _{0 \leq t \leq T}\left|N_{t}(\varphi)\right|^{2}\right] \leq \sup _{n} E^{P_{n}}\left[\sup _{0 \leq t \leq T}\left|N_{t}(\varphi)\right|^{2}\right]<\infty .
$$

Thus it only remains to prove that the expression in (1.19) is a $P$-martingale. Since the analogous expression, with $A_{n}$ replacing $A$ and $B_{n}$ replacing $B$, is a $P_{n}$-martingale, it is easily seen that all we must show is that

$$
E^{P}\left[N_{t}(A \varphi) F\right]=\lim _{n} E^{P^{\left(n^{\prime}\right)}}\left[N_{t}\left(A_{n^{\prime}} \varphi\right) F\right]
$$

for all $t \geq 0, \varphi \in \mathscr{S}\left(R^{d}\right)$, and continnous $F: C\left([0, \infty), \mathscr{H}_{n_{0}}\right) \rightarrow[-1,1]$. But, by (1.6),

$$
E^{p^{(n)}}\left[\left|N_{t}\left(A_{n} \varphi\right)-N_{t}(A \varphi)\right|^{2}\right] \leq t\left\|A_{n} \varphi-A \varphi\right\|^{2}
$$

and clearly $\left\|A_{n} \varphi-A \varphi\right\| \rightarrow 0$ as $n \rightarrow \infty$. Thus proving (1.21) reduces to showing that

$$
E^{P}\left[N_{t}(A \varphi) F\right]=\lim _{n^{\prime}} E^{P^{\left(n^{\prime}\right)}}\left[N_{t}(A \varphi) F\right] .
$$

However $N_{t}(A \varphi) F$ is continuous, and therefore (1.20) together with the assumption that $P^{\left(n^{\prime}\right)} \rightarrow P$ implies (1.22). One final comment is in order. We have just seen that $\left\{P^{(n)}: n \geq 0\right\}$ is precompact and that every limit point satisfies $P\left(N_{0}=0\right)=1$ as well as the hypothesis of Theorem (1.4). Thus by the uniqueness assertion in that theorem, we conclude that $P^{(n)} \rightarrow P$. We summarize our findings in the next 
theorem.

(1.23) Theorem: For each $A$ and $B$ there is a unique probability measure $P$ on $(\Omega, \mathscr{M})$ such that $P\left(N_{0}=0\right)=1$ and $P$ satisfies the hypothesis of Theorem (1.4). Moreover, if $A_{n}=\Pi_{n} A \Pi_{n}, B_{n}=\Pi_{n} B \Pi_{n}$, and $P^{(n)}$ is the measure associated with $A_{n}$ and $B_{n}$, then $P^{(n)}$ tends weakly to $P$.

(1.24) Remark: With very little alteration, the proof just given to get existence can be used to show that if $A_{n}$ and $B_{n}$ are general operators satisfying our basic hypothesis (not necessarily given by $A_{n}=\Pi_{n} A \Pi_{n}$ and $\left.B_{n}=\Pi_{n} B \Pi_{n}\right)$ and if there exists $m_{0} \geq 0$ and $C<\infty$ such that

$$
\sup _{n}\left\|A_{n} \varphi\right\| \leq C\|\varphi\|^{\left(m_{0}\right)}, \quad \varphi \in \mathscr{S}\left(R^{d}\right),
$$

then the measure $P^{(n)}$ associated with $A_{n}$ and $B_{n}$ tends to the $P$ going with $A$ and $B$ if $A_{n} \rightarrow A$ and $B_{n} \rightarrow B$ strongly in $L^{2}\left(R^{d}\right)$.

\section{§2. Branching Brownian Motion Having Finitely Many Particles}

In this section we recast the theory of branching Brownian motion in the setting of Lévy processes. To be precise, let $\left(R^{d}\right)^{(n)}$ be defined for $n \geq 1$ to be the space of equivalence classes of $n$-triples of elements $x \in R^{d} \bmod$ permutations (i. e. $\left\langle x^{1}, \ldots, x^{n}\right\rangle=\left\langle y^{1}, \ldots, y^{n}\right\rangle$ in $\left(R^{d}\right)^{(n)}$ if and only if there is a permutation $\sigma$ such that $y^{k}=x^{\sigma(k)}$, $1 \leq k \leq d)$. Use $\Pi^{(n)}:\left(R^{d}\right)^{n} \rightarrow\left(R^{d}\right)^{(n)}$ to be the map taking $\left(x^{1}, \ldots, x^{n}\right)$ into it's equivalence class in $\left(R^{d}\right)^{(n)}$ and topologize $\left(R^{d}\right)^{(n)}$ so that $\Pi^{(n)}$ is open and continuous. Given a function $f:\left(R^{d}\right)^{(n)} \rightarrow R^{1}$, we say that $f \in C^{k}\left(\left(R^{d}\right)^{(n)}\right)\left(C_{0}^{k}\left(\left(R^{d}\right)^{(n)}\right)\right.$ or $\left.C_{b}^{k}\left(\left(R^{d}\right)^{(n)}\right)\right)$ if $f \circ \Pi^{(n)} \in C^{k}\left(\left(R^{d}\right)^{n}\right)\left(C_{0}^{k}\left(\left(R^{d}\right)^{n}\right.\right.$ or $\left.C_{b}^{k}\left(\left(R^{d}\right)^{n}\right)\right)$. Let $E=\{\phi\} \cup \bigcup_{n=1}^{\infty}\left(R^{d}\right)^{(n)}$, where $\phi$ is an abstract point and topologize $E$ so that $\{\phi\}$ as well as $\left(R^{d}\right)^{(n)}$ for each $n \geq 1$ is both open and closed. Finally, let $\mathscr{D}(E)$ stand for the set of $F: E \rightarrow R^{1}$ such that $F$ restricted to $\left(R^{d}\right)^{(n)}$ is in $C_{0}^{\infty}\left(\left(R^{d}\right)^{(n)}\right)$ for all $n \geq 1$ and 
$F \equiv 0$ on $\left(R^{d}\right)^{(n)}$ for all sufficiently large $n$ 's.

We are now nearly ready to describe the branching Brownian motion with which we will be concerned. However, before doing so, we need a little more notation. First, if $F \in C(E)$ has the property that its restriction $f_{n}$ to $\left(R^{d}\right)^{(n)}$ is in $C^{2}\left(\left(R^{d}\right)^{(n)}\right)$ for all $n \geq 1$, we define $\Delta F(\phi)=0$ and

$$
\Delta F\left(\left\langle x^{1}, \ldots, x^{n}\right\rangle\right)=\sum_{k=1}^{n}\left(\Delta_{k}\left(f_{n} \circ \Pi^{(n)}\right)\right)\left(x^{1}, \ldots, x^{n}\right), \quad n \geq 1,
$$

where $\Delta_{k}$ stands for the Laplacian with respect to $x^{k}$ (notice that $\Delta F$ is indeed well-defined on $E$ ). Next, define for $n \geq 1$ and $1 \leq k \leq n$ $\left\langle x^{1}, \ldots, x^{n}\right\rangle^{k} \in\left(R^{d}\right)^{(n+1)}$ and $\left\langle x^{1}, \ldots, x^{n}\right\rangle_{k} \in\left(R^{d}\right)^{n-1} \quad(\equiv\{\phi\}$ if $n=1)$ to be, respectively, the element of $E$ obtained by repeating or deleting $x^{k}$. With this convention we now define $K$ on $C(E)$ so that $K F(\phi)=0$ and for all $n \geq 1$ :

$$
\begin{aligned}
K F\left(\left\langle x^{1}, \ldots, x^{n}\right\rangle=\sum_{k=1}^{n}(\right. & \frac{F\left(\left\langle x^{1}, \ldots, x^{n}\right\rangle^{k}\right)+F\left(\left\langle x^{1}, \ldots, x^{n}\right\rangle_{k}\right)}{2} \\
& \left.-F\left(\left\langle x^{1}, \ldots, x^{n}\right\rangle\right)\right) .
\end{aligned}
$$

The branching Brownian motion which we want is the Lévy process on $E$ having "diffusion part" determined by $1 / 2 \Delta$ and "jump part" governed by $K$. That is, $\phi$ is absorbing and for $n \geq 1$ the process restricted to $\left(R^{d}\right)^{(n)}$ consists of $n$ indepedent $d$-dimensional Brownian motions, each of which waits a unit exponential holding time and then with equal probabilities splits into two independent copies of itself (moving the process to $\left(R^{d}\right)^{(n+1)}$ ) or disappears (moving the process to $\left.\left(R^{d}\right)^{(n-1)}\right)$.

For our purposes it is best to characterize the above process in terms of a martingale problem. Let $\Omega_{E}=D([0, \infty), E)$ be the space of right continuous functions $[0, \infty)$ into $E$ having left limits, and endow $\Omega_{E}$ with the usual Skorohod topology (this is possible since it is clear that $E$ admits a metrization in which it becomes a Polish space). We will denote a generic element of $E$ by $\eta$ and for $\omega \in \Omega_{E}$ we will use $\eta(t, \omega)$ to denote the position of $\omega$ at time $t \geq 0$. For $t \geq 0$, set $\mathscr{M}_{t}=\sigma(\eta(s): 0 \leq s \leq t)$ and observe that $\sigma\left(\cup_{t \geq 0} \mathscr{M}_{t}\right)$ coincides 
with Borel field $\mathscr{M}$ over $\Omega_{E}{ }^{*}$ Given $\eta \in E$, we say that the probability measure $P$ on $\left(\Omega_{E}, \mathscr{M}\right)$ solves the martingale problem for

$$
\mathscr{L}=1 / 2 \Delta+K
$$

starting from $\eta$ if $P(\eta(0)=\eta)=1$ and

$$
F(\eta(t))-\int_{0}^{t} \mathscr{L} F(\eta(s)) d s
$$

is a $P$-martingale for all $F \in \mathscr{D}(E)$ (i. e. $\left(F(\eta(t))-\int_{0}^{t} \mathscr{L} F(\eta(s)) d s, \mathscr{M}_{t}, P\right.$ ) is a martingale). The following facts are easily deduced from the techniques of $[11]$ :

a) For each $\eta$ there is exactly one solution $P_{\eta}$ to the martingale problem for $\mathscr{L}$ starting from $\eta$ and the family $\left\{P_{\eta}: \eta \in E\right\}$ is Feller continuous and strong Markov.

b) The process $P_{\eta}$ behaves in the manner described in the preceding paragraph.

Alternatively, one can use the results of [5] to arrive at $a$ ) and $b$ ).

For future reference, we will spend the rest of this section deriving some facts and estimates satisfied by the $P_{\eta}$ 's. In the first place, it is not hard to see that if $\widetilde{D}([0, \infty) \times E)$ stands for the class of continuous $F:[0, \infty) \times E \rightarrow C$ such that: $\frac{\partial F}{\partial t} \in C_{b}([0, \infty) \times E)$; the restriction of $F(t, \bullet)$ to $\left(R^{d}\right)^{(n)}, t \geq 0$ and $n \geq 1$, is in $C_{b}^{2}\left(\left(R^{d}\right)^{(n)}\right)$; and $F$ restricted to $[0, \infty) \times\left(R^{d}\right)^{(n)}$ is identically zero for all sufficiently large $n$ 's; then

$$
F(t, \eta(t))-\int_{0}^{t}\left(\frac{\partial F}{\partial s}+\mathscr{L} F\right)(s, \eta(s)) d s
$$

is a $P_{\eta}$-martingale for all $F \in \widetilde{\mathscr{D}}([0, \infty) \times E)$. Next, define $n: E \rightarrow$ $\{0,1, \ldots, n, \ldots\}$ so that

$$
n(\eta)=\left\{\begin{array}{lll}
0 & \text { if } & \eta=\phi \\
n & \text { if } & \eta \in\left(R^{d}\right)^{(n)}
\end{array}\right.
$$

We then have the following estimate.

(2.3) Lemma: If $T>0$ and $\lambda<\log \left(1+\frac{2}{T}\right)$, then 


$$
E^{P_{\eta}}\left[\mathrm{e}^{\lambda n(\eta(T))}\right]=\left(1+\frac{2\left(\mathrm{e}^{\lambda}-1\right)}{2-T\left(e^{\lambda}-1\right)}\right)^{n(\eta)} .
$$

Proof: Set $u(t)=1+\frac{2\left(e^{\lambda}-1\right)}{2-t\left(e^{\lambda}-1\right)}, 0 \leq t \leq T$, and, for $m \geq 1$, define

$$
F_{m}(t, \eta)=\left\{\begin{array}{cl}
(u(t))^{n(\eta)} & \text { if } n(\eta) \leq m \\
0 & \text { if } n(\eta)>m .
\end{array}\right.
$$

It is then clear that

$$
\frac{\partial F_{m}}{\partial s}=\mathscr{L} F_{m}, \quad 0 \leq s \leq T
$$

so long as $n(\eta) \leq m$. Hence

$$
F_{m}\left(T-t \wedge \tau_{m}, \eta\left(t \wedge \tau_{m}\right)\right)
$$

is a $P_{\eta}$-martingale, where

$$
\tau_{m}=(\inf \{t \geq 0: n(\eta(t))>m\}) \wedge T .
$$

In particular,

$$
\begin{gathered}
E^{P_{\eta}}\left[e^{2 n(\eta(T))}\right] \leq E^{P_{\eta}}\left[F_{m}\left(T-\tau_{m}, \eta\left(\tau_{m}\right)\right)\right] \\
\quad=F_{m}(T, \eta)=\left(1+\frac{2\left(e^{\lambda}-1\right)}{2-T\left(e^{\lambda}-1\right)}\right)^{n(\eta)}
\end{gathered}
$$

for all $T>0$ and $\lambda>0$ satisfying $\lambda<\log \left(1+\frac{2}{T}\right)$. From this we see that if $\lambda<\mu<\log \left(1+\frac{2}{T}\right)$, then

$$
E^{P_{\eta}}\left[\left(e^{\lambda n(\eta(t))}\right)^{\mu / \lambda}\right] \leq\left(1+\frac{2\left(e^{\mu}-1\right)}{2-T\left(e^{\mu}-1\right)}\right)^{n(\eta)}, \quad 0 \leq t \leq T
$$

and therefore $\left\{e^{2 n(\eta(t))}: 0 \leq t \leq T\right\}$ are uniformly $P_{\eta}$-integrable. But this means that we can let $m \uparrow \infty$ in the equality

$$
E^{P_{\eta}}\left[F_{m}\left(T-\tau_{m}, n\left(\tau_{m}\right)\right)\right]=\left(1+\frac{2\left(e^{\lambda}-1\right)}{2-T\left(e^{\lambda}-1\right)}\right)^{n(\eta)}
$$

and thereby arrive at (2.4) (since $E^{P_{\eta}}\left[e^{\lambda n(T)}\right]<\infty$ for some $\lambda>0$

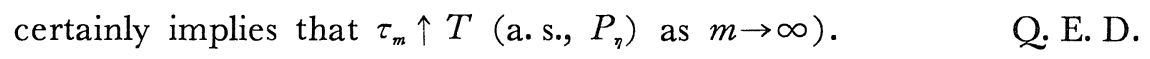

One easy and important consequence of (2.3) is the next theorem. 
(2.5) Theorem: Let $F \in C([0, \infty) \times E)$ have the properties that $\frac{\partial F}{\partial t} \in C([0, \infty) \times E), F$ restricted to $\left(R^{d}\right)^{(n)}$ is in $C_{b}^{2}\left(\left(R^{d}\right)^{(n)}\right)$ for each $n \geq 1$, and there is an $M \geq 0$ and $a C<\infty$ such that

$$
\max \left\{|F(t, \eta)|,\left|\frac{\partial F}{\partial t}(t, \eta)\right|,\left|D^{2} F(t, \eta)\right|\right\} \leq C n(\eta)^{M}
$$

for all $t \geq 0$ and $\eta \in E$, where $D^{2} F(t, \phi) \equiv 0$ and for $\eta \in\left(R^{d}\right)^{(n)}, n \geq 1$ $D^{2} F$ denotes a generic second order spatial derivative of $F_{\circ} \Pi^{(n)}$. Then $f(t, \eta(t))-\int_{0}^{t}\left(\frac{\partial F}{\partial s}+\mathscr{L} F\right)(s, \eta(s)) d s$ is a $P_{\eta}$-martingale for all $\eta \in E$.

(2.6) Corollary: For all $T>0$ and $\lambda<\log \left(1+\frac{2}{T}\right)$

$$
E^{P} \eta\left[\sup _{0 \leq t \leq T} e^{\lambda n(\eta(t))}\right]<\infty
$$

for all $\eta \in E$.

Proof: Take $F(\eta)=n(\eta)$ in the preceding theorem and concluded that $n(\eta(t))$ is a $P_{\eta}$-martingale. Thus, if $\lambda<\log \left(1+\frac{2}{T}\right)$, then $e^{\lambda n(t \wedge T)}$ is a non-negative $P_{\eta}$-submartingale. But this means (by Doob's inequality) that

$$
E^{P_{\eta}}\left[\sup _{0 \leq t \leq T} e^{\lambda n(t)}\right] \leq\left(\frac{\mu / \lambda}{\mu / \lambda-1}\right)^{\mu / \lambda} E^{P_{\eta}}\left[\mathrm{e}^{\mu n(T)}\right]<\infty,
$$

where $\lambda<\mu<\log \left(1+\frac{2}{T}\right)$.

Q. E. D.

At this juncture we want to make a slight change in our point of view. Namely, it is clear that $E$ is homeomorphic to the set of purely atomic integer-valued finite measures on $R^{d}$ given the topology of weak* convergence. That is, given $\eta \in E$ define for $\varphi \in B\left(R^{d}\right)$ :

$$
\eta(\varphi)=\left\{\begin{array}{l}
0 \quad \text { if } \quad \eta=\phi \\
\sum_{1}^{n} \varphi\left(x^{k}\right) \text { if } \eta=\left\langle x^{1}, \ldots, x^{n}\right\rangle, \quad n \geq 1 .
\end{array}\right.
$$

As a consequence of (2.6), we see that for all $\varphi \in B\left(R^{d}\right), \quad T>0$ $\lambda<\log \left(1+\frac{2}{T}\right):$ 


$$
E^{P}\left[\sup _{0 \leq t \leq T} e^{\lambda \eta_{t}(\varphi)}\right]<\infty
$$

where $\eta_{t}(\omega)$ is the measure associated with $\eta(t, \omega)$ by the relation in (2. 7). We are now going to make exact computations of $E^{P_{\eta}}\left[\eta_{t}(\varphi)\right]$ and $E^{p_{\eta}}\left[\left(\eta_{t}(\varphi)\right)^{2}\right]$.

(2.9) Lemma: Set

$$
\gamma_{t}(x)=\frac{1}{(2 \pi t)^{d / 2}} e^{-|x|^{2} / 2 t}, \quad t>0 \text { and } x \in R^{d},
$$

and for $\varphi \in B\left(R^{d}\right)$ define

$$
\varphi_{t}=\gamma_{t} * \varphi
$$

Then

$$
E^{P_{\eta}}\left[\eta_{t}(\varphi)\right]=\eta\left(\varphi_{t}\right), \quad t>0 \text { and } \eta \in E .
$$

Moreover, if

$$
v_{\varphi}(t, \eta)=\eta\left(\left(\varphi^{2}\right)_{t}-\left(\varphi_{t}\right)^{2}+\int_{0}^{t} \gamma_{t-s} *\left(\varphi_{s}\right)^{2} d s\right)+\left(\eta\left(\varphi_{t}\right)\right)^{2}
$$

for $t>0$ and $\eta \in E$, then

$$
E^{P_{\eta}}\left[\eta_{t}^{2}(\varphi)\right]=v_{\varphi}(t, \eta), \quad t>0 \text { and } \eta \in E .
$$

In particular, if $\eta=\langle x\rangle \in\left(R^{d}\right)^{(1)}$, then

$$
E^{P_{\eta}}\left[\eta_{t}^{2}(\varphi)\right]=\left(\varphi^{2}\right)_{t}(x)+\int_{0}^{t}\left[\gamma_{t-s} *\left(\varphi_{s}\right)^{2}\right](x) d s .
$$

Proof: We will assume that $\varphi \in C_{b}^{2}\left(R^{d}\right)$. Given $T>0$, set

$$
F(t, \eta)=\eta\left(\varphi_{T-t}\right), \quad 0 \leq t \leq T
$$

and

$$
G(t, \eta)=v_{\varphi}(T-t, \eta), \quad 0 \leq t \leq T
$$

Since

$$
\frac{\partial F}{\partial t}+\mathscr{L} F=\frac{\partial G}{\partial t}+\mathscr{L} G=0, \quad 0 \leq t \leq T,
$$

it follows from (2.5) that $F(t \wedge T, \eta(t \wedge T))$ and $G(t \wedge T, \eta(t \wedge T))$ are $P_{\eta}$-martingales. Thus 


$$
E^{P_{\eta}}\left[\eta_{T}(\varphi)\right]=E^{P_{\eta}}[F(T, \eta(T))]=F(0, \eta)=\eta\left(\varphi_{T}\right)
$$

and

$$
E^{P_{\eta}}\left[\eta_{T}^{2}(\varphi)\right]=E^{P_{\eta}}[G(T, \eta(T))]=G(0, \eta)=v_{\varphi}(T, \eta) .
$$

Q. E. D.

For reasons which will not become apparent until the next section, we need one more estimate.

\section{(2.15) Lemma: Set}

$$
\Psi(x)=\left(1+|x|^{d+1}\right)^{-1}, \quad x \in R^{d} .
$$

Then for each $T>0$ there exists a constant $A(T)<\infty$ such that

$$
E^{P}\langle x\rangle\left[\sup _{0 \leq t \leq T} \eta_{t}^{2}(\Psi)\right] \leq A(T) \Psi^{2}(x), \quad x \in R^{d} .
$$

Proof: For each $T>0$ it is elementary to see that there exist constants $C_{1}(T), C_{2}(T)$, and $C_{3}(T)<\infty$ such that

$$
\frac{1}{C_{1}(T)} \Psi_{t} \leq \Psi \leq C_{2}(T) \Psi_{t}, \quad 0 \leq t \leq T,
$$

and

$$
\left(\Psi^{2}\right)_{t} \leq C_{3}(T) \Psi^{2}, \quad 0 \leq t \leq T
$$

Since, by (2.5), $\eta_{t \wedge T}\left(\varphi_{T-t \wedge T}\right)$ is a $P_{\langle x\rangle}$-martingale, we have by Doob's inequality and (2.14):

$$
\begin{aligned}
& E^{P\langle x\rangle}\left[\sup _{0 \leq t \leq T} \eta_{t}^{2}\left(\Psi_{T-t}\right)\right] \leq 4 E^{P\langle x\rangle}\left[\eta_{T}^{2}(\Psi)\right] \\
& \quad=4\left(\Psi^{2}\right)_{T}(x)+4 \int_{0}^{T}\left[\gamma_{T-s} *\left(\Psi_{s}\right)^{2}\right](x) d s \\
& \quad \leq 4 C_{3}(T) \Psi^{2}(x)+4\left(C_{1}(T)\right)^{2} \int_{0}^{T}\left[\gamma_{T-s} *(\Psi)^{2}\right](x) d s \\
& \quad \leq 4\left(C_{3}(T)+\left(C_{1}(T)\right)^{2} C_{3}(T) T\right) \Psi^{2}(x) .
\end{aligned}
$$

At the same time, $\eta_{t}^{2}(\Psi) \leq C_{2}(T) \eta_{t}^{2}\left(\Psi_{T-t}\right)$. Combining this with the preceding, we arrive at (2.17).

Q. E. D. 


\section{§ 3. Branching Brownian Motion Having Infinitely Many Particles}

In this section we again discuss processes of the sort introduced in section (2), only now we allow there to be infinitely many particles initially. The approach that we adopt mimicks, for our setting, the construction by Durrett [3].

For each $x \in R^{d}$ let $\Omega_{x}=\Omega_{E}$ and $\mathscr{M}_{x}=\mathscr{M}$. Given a probability space $(\bar{\Omega}, \overline{\mathscr{M}}, \bar{P})$ on which there is an $R^{d}$-valued point process $\bar{\eta}$ satisfying

$$
E^{p}[\operatorname{card}(\Gamma \cap \bar{\eta})]=|\Gamma|
$$

for bounded $\Gamma \in \mathscr{B} R^{d}(|\Gamma|$ denotes the Lebesgue measure of $\Gamma$ ), let $\tilde{P}=\bar{P} \times \prod_{x \in R^{d}} P_{x}$ on $(\tilde{Q}, \tilde{\mathscr{M}})$, where $\tilde{\Omega}=\bar{\Omega} \times \prod_{x \in R^{d}} \Omega_{x}$ and $\tilde{\mathscr{M}}=\overline{\mathscr{M}} \times \prod_{x \in R^{d}} \mathscr{M}_{x}$. If $\tilde{\omega} \in \tilde{\Omega}$, we let $\omega_{x}, x \in R^{d}$, denote the $x^{t h \in R^{d}}$ coordinate of $\tilde{\omega}$ and define $\eta_{t, x}(\tilde{\omega})=\eta_{t}\left(\omega_{x}\right)$.

(3.2) Lemma: Let $\Psi$ be the function in (2.16). Then for all $T>0$ :

$$
E^{\tilde{P}}\left[\sum_{x \in \eta} \sup _{0 \leq t \leq T} \eta_{t, x}(\Psi)\right]<\infty
$$

In particular, if

$$
\tilde{\eta}_{t}=\sum_{x \in \eta} \eta_{t, x}
$$

then there is a $\tilde{P}$-null set $B \in \tilde{M}$ such that for all $\tilde{\omega} \notin B$ the function $t \rightarrow \tilde{\eta}_{t}(\tilde{\omega})$ is right continuous and has left limits as a mapping from $[0, \infty)$ into $\mathscr{S}^{\prime}\left(R^{d}\right)$.

Proof: Suppose that (3.3) has been proved. Then there is a $\tilde{P}$-null set $B \in \tilde{\mathscr{M}}$ such that

$$
\sum_{x \in \eta} \eta_{t, x}(\Psi, \tilde{\omega})
$$

converges uniformly on each bounded time interval for each $\tilde{\omega} \notin B$. But if $\varphi \in \mathscr{S}\left(R^{d}\right)$, then $|\varphi| \leq C_{\varphi} \Psi$ for some $C_{\varphi}<\infty$, and therefore for 
each $\tilde{\omega} \notin B \quad \sum_{x \in \eta} \eta_{t, x}(\varphi, \tilde{\omega})$ converges uniformly and absolutely on each bounded time interval. Since $\eta_{t, x}(\varphi, \tilde{\omega})$ is right continuous and has left limits for all $x \in R^{d}, \varphi \in \mathscr{S}\left(R^{d}\right)$, and $\tilde{\omega} \in \tilde{Q}$, we conclude that for all $\omega \notin B t \rightarrow \tilde{\eta}_{t}(\tilde{\omega})$ has the desired properties.

To prove (3.3), observe that by (2.17):

$$
E^{r}\left[\sup _{0 \leq t \leq T} \eta_{t, x}(\Psi)\right] \leq C^{1 / 2}(T) \Psi(x) .
$$

Thus, by (3. 1) :

$$
\begin{aligned}
E^{p}\left[\sum_{x \in \eta} \sup _{0 \leq t \leq T} \eta_{t, x}(\Psi)\right] & \leq C^{1 / 2}(T) E^{p}\left[\sum_{x \in \eta} \Psi(x)\right] \\
& =C^{1 / 2}(T) \int \Psi(x) d x<\infty .
\end{aligned}
$$

Q. E. D.

Define $\tilde{\mathscr{M}}_{t}$ for $t \geq 0$ to be the $\sigma$-algebra of subsets of $\tilde{\Omega}$ generated by $\tilde{\eta}_{s}(\varphi)$ for $0 \leq s \leq t$ and $\varphi \in \mathscr{S}\left(R^{d}\right)$. The next theorem plays a crucial role in our future results.

(3.5) Theorem: Given $f \in C_{b}^{2}\left(R^{1}\right)$ and $\varphi \in \mathscr{S}\left(R^{d}\right)$,

(3.6) $f\left(\tilde{\eta}_{t}(\varphi)\right)-\int_{0}^{t} \tilde{\eta}_{s}(1 / 2 \Delta \varphi) f^{\prime}\left(\tilde{\eta}_{s}(\varphi)\right) d s$

$$
\begin{aligned}
& -1 / 2 \int_{0}^{t} \tilde{\eta}_{s}\left(|\nabla \varphi|^{2}\right) f^{\prime \prime}\left(\tilde{\eta}_{s}(\varphi)\right) d s \\
& -\int_{0}^{t} \tilde{\eta}_{s}\left(\frac{f\left(\tilde{\eta}_{s}(\varphi)+\varphi(y)\right)+f\left(\tilde{\eta}_{s}(\varphi)-\varphi(y)\right)}{2}\right. \\
& \left.\quad-f\left(\tilde{\eta}_{s}(\varphi)\right)\right) d s
\end{aligned}
$$

is an $\left(\tilde{\Omega}, \tilde{M}_{t}, \tilde{P}\right)$-martingale.

Proof: What we must show is that if $0 \leq t_{1}<t_{2}$ then

$$
E^{\vec{p}}\left[X\left(t_{2}\right)-X\left(t_{1}\right), B\right]=0
$$

where

$$
\begin{aligned}
X(t)=f\left(\tilde{\eta}_{t}(\varphi)\right) & -\int_{0}^{t} \tilde{\eta}_{s}(1 / 2 \Delta \varphi) f^{\prime}\left(\tilde{\eta}_{s}(\varphi)\right) d s \\
& -1 / 2 \int_{0}^{t} \tilde{\eta}_{s}\left(|\nabla \varphi|^{2}\right) f^{\prime \prime}\left(\tilde{\eta}_{s}(\varphi)\right) d s \\
& -\int_{0}^{t} \tilde{\eta}_{s}\left(\frac{f\left(\tilde{\eta}_{s}(\varphi)+\varphi(y)\right)+f\left(\tilde{\eta}_{s}(\varphi)-\varphi(y)\right)}{2}\right.
\end{aligned}
$$




$$
\left.-f\left(\tilde{\eta}_{s}(\varphi)\right)\right) d s
$$

and $B$ is a set of the form

$$
\left\{\tilde{\eta}_{s_{1}}\left(\varphi_{1}\right) \in \Gamma_{1}, \ldots, \tilde{\eta}_{s_{n}}\left(\varphi_{n}\right) \in \Gamma_{n}\right\}
$$

with $n \geq 1 ; 0 \leq s_{1} \leq \ldots \leq s_{n} \leq t_{1} ; \varphi_{1}, \ldots, \varphi_{n} \in \mathscr{S}\left(R^{d}\right) ;$ and $\Gamma_{1}, \ldots, \Gamma_{n} \in \mathscr{B} R^{d}$. Given $m \geq 1$, set

$$
\tilde{\eta}_{t}^{(m)}=\sum_{\substack{x \in \in_{j} \\|x| \leq m}} \eta_{t, x}
$$

and define $X^{(m)}(\bullet)$ in terms of $f, \varphi$, and $\tilde{\eta}^{(m)}$. the same way as $X(\bullet)$ is given in terms of $f$, $\varphi$, and $\tilde{\eta}$. Then

$$
\begin{aligned}
& \left.E^{\tilde{P}}\left[X^{(m)} t_{2}\right)-X^{(m)}\left(t_{1}\right), B\right]=
\end{aligned}
$$

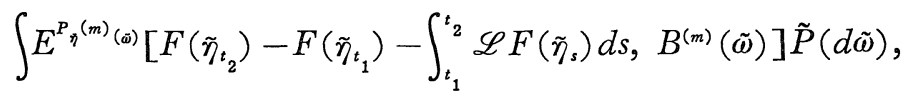

where

$$
\begin{aligned}
& \bar{\eta}^{(m)}=\bar{\eta} \cap\{x:|x| \leq m\}, \\
& F(\eta)=f(\eta(\varphi)),
\end{aligned}
$$

and

$$
B^{(m)}(\tilde{\omega})=\left\{\eta_{s_{j}}^{(m)}\left(\varphi_{j}\right)+\sum_{\substack{x \in \eta^{(\tilde{\omega})} \\|x|>m}} \eta_{s_{j}, x}\left(\varphi_{j}, \tilde{\omega}\right) \in \Gamma_{j}, 1 \leq j \leq n\right\}
$$

Since (2.5) applies to $F$, we know that

$$
E^{P_{\eta}^{(m)}(\tilde{\omega})}\left[F\left(\tilde{\eta}_{t_{2}}\right)-F\left(\tilde{\eta}_{t_{1}}\right)-\int_{t_{1}}^{t_{2}} \mathscr{L} F\left(\tilde{\eta}_{s}\right) d s, B^{(m)}(\tilde{\omega})\right]=0
$$

for each $\tilde{\omega}$, and therefore we have now shown that

$$
\left(X^{(m)}(t), \tilde{M}_{t}, \tilde{P}\right)
$$

is a martingale. But, using (3.3), it is easy to check that

$$
X^{(m)}(t) \rightarrow X(t) \text { as } m \rightarrow \infty \text { in } L^{1}(P) .
$$

Q. E. D.

We conclude this section with some preparations for the next two sections. From now on we will be making the following assumption about $\bar{\eta}$ (in addition to (3.1)) : 


$$
\left.E^{p}\left[\bar{\eta}(\varphi)-\int \varphi(x) d x\right)^{2}\right] \leq C\|\varphi\|^{2}, \varphi \in \mathscr{S}\left(R^{d}\right),
$$

for some $1 \leq C<\infty$. Notice that both (3.1) and (3.7) are satisfied by a Poisson random field. Also, observe that, in view of (3.1), (3.7) is an estimate on the variance of $\bar{\eta}(\varphi)$.

(3. 8) Lemma: For all $t>0$ and $\varphi \in \mathscr{S}\left(R^{d}\right)$ :

$$
E^{\tilde{P}}\left[\tilde{\eta}_{t}(\varphi)\right]=\int \varphi(x) d x
$$

and

$$
E^{\tilde{P}}\left[\left(\tilde{\eta}_{t}(\varphi)-\int \varphi(x) d x^{2}\right] \leq(C+t)\|\varphi\|^{2}\right.
$$

Proof: To see (3.9), note that by (3.1) and (2.12):

$$
\begin{aligned}
E^{\tilde{P}}\left[\eta_{t}(\varphi)\right] & =E^{\tilde{P}}\left[\sum_{x \in \eta} \eta_{t, x}(\varphi)\right] \\
& =E^{\tilde{P}}\left[\bar{\eta}\left(\varphi_{t}\right)\right]=\int \varphi(x) d x .
\end{aligned}
$$

Next, by (2.12) and (2.14):

$$
\begin{aligned}
& \left.E^{\tilde{P}}\left[\tilde{\eta}_{t}(\varphi)\right)^{2}\right]=E^{\tilde{P}}\left[\left(\sum_{x \in \tilde{\eta}} \eta_{t, x}(\varphi)\right)^{2}\right] \\
& =E^{\tilde{P}}\left[\sum_{x \in \eta}\left(\eta_{t, x}(\varphi)\right)^{2}\right]+E^{\tilde{P}}\left[\sum_{\substack{x, y \in \eta \\
x \neq y}} \eta_{t, x}(\varphi) \eta_{t, y}(\varphi)\right] \\
& =E^{\tilde{p}}\left[\bar{\eta}\left(\left(\varphi^{2}\right)_{t}\right)\right]+\int_{0}^{t} E^{\bar{p}}\left[\bar{\eta}\left(\gamma_{t-s} *\left(\varphi_{s}\right)^{2}\right)\right] d s \\
& +E^{p}\left[\sum_{\substack{x, y \in \eta \\
x \neq y}} \varphi_{t}(x) \varphi_{t}(y)\right] \\
& =E^{p}\left[\bar{\eta}\left(\left(\varphi^{2}\right)_{t}\right)\right]+\int_{0}^{t} E^{p}\left[\bar{\eta}\left(\gamma_{t-s} *\left(\varphi_{s}\right)^{2}\right)\right] d s \\
& +E^{p}\left[\left(\bar{\eta}\left(\varphi_{t}\right)\right)^{2}\right]-E^{p}\left[\bar{\eta}\left(\left(\varphi_{t}\right)^{2}\right)\right],
\end{aligned}
$$

so

$$
\begin{aligned}
E^{\tilde{P}}\left[\left(\tilde{\eta}_{t}(\varphi)\right.\right. & \left.\left.-\int \varphi(x) d x\right)^{2}\right] \\
& \leq C\left\|\varphi_{t}\right\|^{2}+\|\varphi\|^{2}+\int_{0}^{t}\left\|\varphi_{s}\right\|^{2} d s-\left\|\varphi_{t}\right\|^{2} \\
& \leq(C-1)\left\|\varphi_{t}\right\|^{2}+(1+t)\|\varphi\|^{2}
\end{aligned}
$$


We next introduce a "central limit" type scaling of the process $\tilde{\eta}$. Given $\alpha>0$, we set

$$
\varphi^{(\alpha)}(x)=\frac{\varphi(x / \alpha)}{\alpha^{(d+2) / 2}}
$$

and define

$$
\eta_{t}^{(\alpha)}(\varphi)=\tilde{\eta}_{\alpha^{2} t}\left(\varphi^{(\alpha)}\right)-\int \varphi^{(\alpha)}(x) d x
$$

Observe that

$$
E^{\tilde{P}}\left[\eta_{t}^{(\alpha)}(\varphi)\right]=0
$$

and

$$
\begin{aligned}
\left.E^{\tilde{P}}\left[\eta_{t}^{(\alpha)}(\varphi)\right)^{2}\right] & =E^{\vec{P}}\left[\left(\tilde{\eta}_{\alpha^{2} t}\left(\varphi^{(\alpha)}\right)-\int \varphi^{(\alpha)}(x) d x\right)^{2}\right] \\
& \leq\left(C+\alpha^{2} t\right)\left\|\varphi^{(\alpha)}\right\|^{2}=\left(\frac{C}{\alpha^{2}}+t\right)\|\varphi\|^{2} .
\end{aligned}
$$

That is :

$$
E^{\bar{P}}\left[\left(\eta_{t}^{(\alpha)}(\varphi)\right)^{2}\right] \leq\left(\frac{C}{\alpha^{2}}+t\right)\|\varphi\|^{2}
$$

We next want to see what must be subtracted from $f\left(\tilde{\eta}_{t}^{(\alpha)}(\varphi)\right)$ to get a $\tilde{P}$-martingale. First observe that by (3.5) for $f \in C^{2}\left(R^{1}\right)$

$$
\begin{gathered}
f\left(\tilde{\eta}_{\alpha^{2} t}\left(\varphi^{(\alpha)}\right)\right)-\frac{1}{2} \int_{0}^{\alpha^{2} t} \tilde{\eta}_{s}\left(\left|\nabla \varphi^{(\alpha)}\right|^{2}\right) f^{\prime \prime}\left(\tilde{\eta}_{s}\left(\varphi^{(\alpha)}\right)\right) d s \\
-\frac{1}{2} \int_{0}^{t} \tilde{\eta}_{s}\left((\Delta \varphi)^{(\alpha)}\right) f^{\prime}\left(\tilde{\eta}_{s}\left(\varphi^{(\alpha)}\right)\right) d s \\
-\int_{0}^{a^{2} t} d s \int\left[\frac{f\left(\tilde{\eta}_{s}\left(\varphi^{(\alpha)}\right)+\varphi^{(\alpha)}(y)\right)+f\left(\tilde{\eta}_{s}\left(\varphi^{(\alpha)}\right)-\varphi^{(\alpha)}(y)\right)}{2}\right. \\
\left.-f\left(\tilde{\eta}_{s}^{(a)}(\varphi)\right)\right] \tilde{\eta}_{s}(d y)
\end{gathered}
$$

is an $\left(\tilde{\Omega}, \tilde{\mathscr{M}}_{a^{2} t}, \tilde{P}\right)$ martingale. Since

$$
\left|\nabla \varphi^{(\alpha)}\right|^{2}(x)=\alpha^{-(d+6) / 2}\left(|\nabla \varphi|^{2}\right)^{(\alpha)}(x)
$$

and 


$$
\Delta \varphi^{(\alpha)}(x)=\alpha^{-2}(\Delta \varphi)^{(\alpha)}(x),
$$

we conclude that

$$
\begin{gathered}
f\left(\tilde{\eta}_{\alpha^{2} t}\left(\varphi^{(\alpha)}\right)\right)-\frac{\alpha^{-(d+2) / 2}}{2} \int_{0}^{t} \tilde{\eta}_{\alpha^{2} s}\left(\left(|\nabla \varphi|^{2}\right)^{(\alpha)}\right) f^{\prime \prime}\left(\tilde{\eta}_{\alpha^{2} s}\left(\varphi^{(\alpha)}\right)\right) d s \\
-\int_{0}^{t} \tilde{\eta}_{\alpha^{2} s}\left((1 / 2 \Delta \varphi)^{(\alpha)}\right) f^{\prime}\left(\tilde{\eta}_{\alpha^{2} s}\left(\varphi^{(\alpha)}\right)\right) d s \\
-\alpha^{2} \int_{0}^{t} \tilde{\eta}_{\alpha^{2} s}\left(\frac{\left(f\left(\tilde{\eta}_{\alpha^{2} s}\left(\varphi^{(\alpha)}\right)+\varphi^{(\alpha)}(\cdot)\right)+f\left(\tilde{\eta}_{\alpha^{2} s}\left(\varphi^{(\alpha)}\right)-\varphi^{(\alpha)}(\cdot)\right)\right.}{2}\right. \\
\left.-f\left(\tilde{\eta}_{\alpha^{2} s}\left(\varphi^{(\alpha)}\right)\right)\right) d s
\end{gathered}
$$

is an $\left(\tilde{\Omega}, \tilde{\mathscr{M}}_{\alpha^{2} t}, \tilde{P}\right)$ martingale. Finally, because $\int \Delta \psi(x) d x=0$ for all $\phi \in \mathscr{S}\left(R^{d}\right)$, we now have that for all $f \in C_{b}^{2}\left(R^{d}\right)$ :

$$
\begin{aligned}
f\left(\eta_{t}^{(\alpha)}(\varphi)\right)-\frac{\alpha^{-(d+2) / 2}}{2} \int_{0}^{t} \tilde{\eta}_{\alpha_{s}{ }_{s}}\left(\left(|\nabla \varphi|^{2}\right)^{(\alpha)}\right) f^{\prime \prime}\left(\eta_{s}^{(\alpha)}(\varphi)\right) d s \\
-\int_{0}^{t} \eta_{s}^{(\alpha)}(1 / 2 \Delta \varphi) f^{\prime}\left(\eta_{s}^{(\alpha)}(\varphi)\right) d s \\
-\alpha^{2} \int_{0}^{t} \tilde{\eta}_{\alpha_{s}{ }_{s}}\left(\frac{f\left(\eta_{s}^{(\alpha)}(\varphi)+\varphi^{(\alpha)}(\cdot)\right)+f\left(\eta_{s}^{(\alpha)}(\varphi)-\varphi^{(\alpha)}(\cdot)\right)}{2}\right. \\
\left.-f\left(\eta_{s}^{(\alpha)}(\varphi)\right)\right) d s
\end{aligned}
$$

is an $\left(\tilde{\Omega}, \tilde{\mathscr{M}}_{\alpha^{2} t}, \tilde{P}\right)$ martingale for all $f \in C_{b}^{2}\left(R^{1}\right)$ and $\varphi \in \mathscr{S}\left(R^{d}\right)$.

Our final task in this section is to estimate $E^{\tilde{P}}\left[\sup _{0 \leq t \leq T}\left(\eta_{t}^{(\alpha)}(\varphi)\right)^{2}\right]$. To this end first observe that (3.14) together with (3.15) shows that

$$
\eta_{t}^{(\alpha)}(\varphi)-\int_{0}^{t} \eta_{s}^{(\alpha)}(1 / 2 \Delta \varphi) d s
$$

is an $\left(\tilde{Q}, \widetilde{\mathscr{M}}_{\alpha^{2} t}, \tilde{P}\right)$ martingale. Thus, by Doob's inequality

$$
\begin{aligned}
E^{\bar{P}}\left[\sup _{0 \leq t \leq T}\left(\eta_{t}^{(\alpha)}(\varphi)-\int_{0}^{t} \eta_{s}^{(\alpha)}(1 / 2 \Delta \varphi) d s\right)^{2}\right]^{1 / 2} \\
\quad \leq 2 E^{\tilde{P}}\left[\left(\eta_{T}^{(\alpha)}(\varphi)-\int_{0}^{T} \eta_{s}^{(\alpha)}(1 / 2 \Delta \varphi) d s\right)^{2}\right]^{1 / 2} \\
\quad \leq 2 E^{\tilde{P}}\left[\left(\eta_{T}^{(\alpha)}(\varphi)\right)^{2}\right]^{1 / 2}+2 E^{\tilde{P}}\left[\left(\int_{0}^{T} \eta_{s}^{(\alpha)}(1 / 2 \Delta \varphi) d s\right)^{2}\right]^{1 / 2} \\
\quad \leq 2\left(\frac{C}{\alpha^{2}}+T\right)^{1 / 2}\left(\|\varphi\|+T\left(\frac{C}{\alpha^{2}}+T\right)^{1 / 2}\|\Delta \varphi\|\right),
\end{aligned}
$$

and therefore : 


$$
\begin{aligned}
E^{\tilde{P}}\left[\sup _{0 \leq t \leq T}\left(\eta_{t}^{(\alpha)}(\varphi)\right)^{2}\right]^{1 / 2} & \leq E^{\hat{P}}\left[\sup _{0 \leq t \leq T}\left(\eta_{t}^{(\alpha)}(\varphi)-\int_{0}^{t} \eta_{s}^{(\alpha)}(1 / 2 \Delta \varphi) d s\right)^{2}\right]^{1 / 2} \\
& +E^{\tilde{P}}\left[\sup _{0 \leq t \leq T}\left(\int_{0}^{t} \eta_{s}^{(\alpha)}(1 / 2 \Delta \varphi) d s\right)^{2}\right]^{1 / 2} \\
& \leq 2\left(\frac{C}{\alpha^{2}}+T\right)^{1 / 2}(\|\varphi\|+T\|\Delta \varphi\|) .
\end{aligned}
$$

We have therefore proved that

$$
E^{\tilde{P}}\left[\sup _{0 \leq t \leq T}\left(\eta_{t}^{(\alpha)}(\varphi)\right)^{2}\right] \leq 8\left(\frac{C}{\alpha^{2}}+T\right)\left(\|\varphi\|^{2}+T^{2}\|\Delta \varphi\|^{2}\right) .
$$

Once one has (3.16), it is easy to extend (3.15) to the function $f(x)=x^{2}$ and thereby conclude that

$$
\begin{aligned}
\left(\eta_{t}^{(\alpha)}(\varphi)\right)^{2} & -\alpha^{-(d+2) / 2} \int_{0}^{t} \tilde{\eta}_{a_{s}}\left(\left.(\mid \nabla \varphi)^{2}\right|^{(\alpha)}\right) d s \\
& -\int_{0}^{t} \eta_{s}^{(\alpha)}(\Delta \varphi) \eta_{s}^{(\alpha)}(\varphi) d s \\
& -\alpha^{2} \int_{0}^{t} \tilde{\eta}_{\alpha^{2} s}\left(\left(\varphi^{(\alpha)}\right)^{2}\right) d s
\end{aligned}
$$

is an $\left(\hat{Q}, \tilde{\mathscr{M}}_{\alpha^{2} t}, \tilde{P}\right)$ martingale. If we now set

$$
\tilde{\mu}_{t}^{(\alpha)}(\varphi)=\eta_{t}^{(\alpha)}(\varphi)-\int_{0}^{t} \eta_{s}^{(\alpha)}(1 / 2 \Delta \varphi) d s
$$

then

$$
\begin{aligned}
\left(\tilde{\mu}_{t}^{(\alpha)}(\varphi)\right)^{2} & +\left(\int_{0}^{t} \eta_{s}^{(\alpha)}(1 / 2 \Delta \varphi) d s\right)^{2} \\
& =\left(\eta_{t}^{(\alpha)}(\varphi)\right)^{2}-2 \tilde{\mu}_{t}^{(\alpha)}(\varphi) \int_{0}^{t} \eta_{s}^{(\alpha)}(1 / 2 \Delta \varphi) d s
\end{aligned}
$$

Combining the martingale involving $\left(\eta_{t}^{(\alpha)}(\varphi)\right)^{2}$ with the fact that $\tilde{\mu}_{t}^{(\alpha)}(\varphi)$ and therefore (cf. Lemma 2.1 in [12])

$$
\tilde{\mu}_{t}^{(\alpha)}(\varphi) \int_{0}^{t} \eta_{s}^{(\alpha)}(1 / 2 \Delta \varphi) d s-\int_{0}^{t} \tilde{\mu}_{s}^{(\alpha)}(\varphi) \eta_{s}^{(\alpha)}(1 / 2 \Delta \varphi) d s
$$

are $\left(\tilde{\Omega}, \tilde{\mathscr{M}}_{\alpha^{2} t}, \tilde{P}\right)$ martingales, we arrive at the conclusion that

$$
\left(\tilde{\mu}_{t}^{(\alpha)}(\varphi)\right)^{2}-\alpha^{-(d+2) / 2} \int_{0}^{t} \tilde{\eta}_{\alpha^{2} s}\left(\left(|\nabla \varphi|^{2}\right)^{(\alpha)}\right) d s-\alpha^{2} \int_{0}^{t} \tilde{\eta}_{\alpha^{2} s}\left(\left(\varphi^{(\alpha)}\right)^{2}\right) d s
$$

is an $\left(\tilde{Q}, \tilde{\mathscr{M}}_{\alpha^{2} t}, \tilde{P}\right)$ martingale. Equivalently, 


$$
\begin{gathered}
\left(\tilde{\mu}_{t}^{(\alpha)}(\varphi)\right)^{2}-\alpha^{-(d+2) / 2} \int_{0}^{t} \eta_{s}^{(\alpha)}\left(|\nabla \varphi|^{2}\right) d s-\alpha^{-(d-2) / 2} \int_{0}^{t} \eta_{s}^{(\alpha)}\left(\varphi^{2}\right) d s \\
-\alpha^{-2} t\||\nabla \varphi|\|^{2}-t \|\left.\varphi\right|^{2}
\end{gathered}
$$

is an $\left(\tilde{\Omega}, \tilde{\mathscr{M}}_{\alpha^{2} t}, \tilde{P}\right)$ martingale.

\section{§4. Tightness of the Processes $\tilde{\eta}^{(\alpha)}$}

Let $(\tilde{\Omega}, \tilde{\mathscr{M}}, \tilde{P}),\left\{\tilde{\mathscr{M}}_{t}: t \geq 0\right\}$, and $\eta^{(\alpha)}$. be as in section (3) and denote by $P^{(\alpha)}$ the distribution on $D\left([0, \infty), \mathscr{S}^{\prime}\left(R^{d}\right)\right)$ of $\eta^{(\alpha)}$. under $\tilde{P}$ (cf. Lemma (3.2)). What we are going to do in this section is show that $\left\{P^{(\alpha)}: \alpha \geq 1\right\}$ is a tight (i. e. pre-compact) family, and then we will show that for $d \geq 3$ the limit $\lim _{\alpha \rightarrow \infty} P^{(\alpha)}$ exists and is the OrnsteinUhlenbeck process with characteristics $1 / 2 \Delta$ and $I$. Although it is in conflict with the notation in section (1), we will use in this section $\Omega$ to denote $D\left([0, \infty), \mathscr{S}^{\prime}\left(R^{d}\right)\right)$ and then define $N_{t}, t \geq 0 ; \mathscr{M}$; and $\left\{\mathscr{M}_{t}: t \geq 0\right\}$ correspondly as in section (1) (only now for our new choice of $\Omega$ ). Finally, define the Hilbert space $\mathscr{H}_{d+3}$ with norm $\|\cdot \cdot\| \mid$ as in (1.16) and (1.17) (i. e. take $n_{0}=d+3$ in those equations). The next lemma shows that the $P^{(\alpha)}$ are concentrated on $D\left([0, \infty), \mathscr{H}_{d+3}\right)$.

(4.1) Lemma: If $T>0$ and $\alpha \geq 1$, then

$$
E^{p^{(\alpha)}}\left[\sup _{0 \leq t \leq T}\left(N_{t}\left(h_{\beta}\right)\right)^{2}\right] \leq 8(C+T)\left(1+T^{2}(2|\beta|+d)^{2}\right)
$$

In particular, for all $T>0$ :

$$
\sup _{\alpha \geq 1} E^{P^{(\alpha)}}\left[\sup _{0 \leq t \leq T}\left\|! \mid N_{t}\right\| \|^{2}\right]<\infty
$$

and

$$
\lim _{n \rightarrow \infty} \sup _{\alpha \geq 1} E^{P^{(\alpha)}}\left[\sup _{0 \leq t \leq T}\left\|\mid \Pi_{n}^{\perp} N_{t}\right\|^{2}\right]=0
$$

where $\Pi_{n}$ is defined as in section (1).

Proof: Clearly all that we have to do is prove (4.2). But (4.2) is an immediate consequence of (3.16) plus the inequality (cf. A. 9)

$$
\|\left.\Delta h_{\beta}\right|^{2} \leq(2|\beta|+d)^{2} \quad \text { Q. E. D. }
$$


In view of (4.4) we will have shown that $\left\{P^{(\alpha)}: \alpha \geq 1\right\}$ is tight and that any limit, as $\alpha \rightarrow \infty$, lives on $C\left([0, \infty), \mathscr{H}_{d+3}\right)$ once we show that for all $n \geq 1, \varepsilon>0$, and $T>0$ there is an $\alpha_{0}$ and a $\delta>0$ for which:

$$
\sup _{\alpha \geq \alpha_{0}} P^{(\alpha)}\left(\sup _{\substack{0 \leq \leq \leq \leq \leq T \\ t \in s<\delta}}\left\|\Pi_{n} N_{t}-\Pi_{n} N_{s}\right\| \geq \varepsilon\right) \leq \varepsilon .
$$

Since $I_{n} N$. is finite-dimensional, this reduces to studying each coordinate $N_{.}\left(h_{\beta}\right),|\beta| \leq n$, separately. Precisely what we need is proved in the next lemma.

(4.6) Lemma: For all $\beta, \varepsilon>0$ and $T>0$ there is an $\alpha_{0} \geq 1$ and a $\delta>0$ such that

$$
\left.\sup _{\alpha \geq \alpha_{0}} P^{(\alpha)} \sup _{\substack{0 \leq s \leq t \leq T \\ t \leq s<\delta}}\left|N_{t}\left(h_{\beta}\right)-N_{s}\left(h_{\beta}\right)\right|>\varepsilon\right) \leq \varepsilon
$$

Proof: Given any right-continuous function $f:[0, T] \rightarrow R^{1}$ having left limits define

$$
\begin{aligned}
\tilde{\omega}_{f}(\delta)= & \sup _{\substack{0 \leq t_{1} \leq t_{2} \leq t_{3} \\
\left(t_{3}-t_{2}\right) \vee\left(t_{2}-t_{1}\right)<\delta}}\left\{\left|f\left(t_{3}\right)-f\left(t_{2}\right) ! \wedge\right| f\left(t_{2}\right)-f\left(t_{1}\right) \mid\right\} \bigvee \\
& \left(\sup _{0 \leq t \leq \delta}|f(t)-f(0)|\right) \bigvee\left(\sup _{0 \leq t \leq \delta}|f(T)-f(T-t)|\right)
\end{aligned}
$$

and

$$
\omega_{f}(\delta)=\sup _{\substack{0 \leq t_{1}<t_{2} \leq T \\ t_{2}-t_{1}<\delta}}\left|f\left(t_{2}\right)-f\left(t_{1}\right)\right| .
$$

Under the assumption that $\sup _{0 \leq t \leq T}|f(t+)-f(t-)| \leq \gamma$, it is well-known (cf. Parthasarathy [10] Lemma 6. 4) that $\omega_{f}(\delta) \leq 2 \tilde{\omega}_{f}(\delta)+\gamma$. Thus in order to prove (4.7), all that we have to do is choose $\alpha_{0} \geq 1$ so that $\sup _{x}\left|h_{\beta}^{(\alpha)}(x)\right|<\varepsilon / 2$ whenever $\alpha \geq \alpha_{0}$ and then show that

$$
\lim _{\delta \rightarrow 0} \sup _{\alpha \geq \alpha_{0}} P^{(\alpha)}\left(\tilde{\omega}_{. N\left(h_{\beta}\right)}(\delta) \geq \varepsilon / 4\right)=0 .
$$

The reason that this suffices is because one can easily show (cf. [11]) from (3.15) that

$$
P^{(\alpha)}\left(\left|N_{t+}(\varphi)-N_{t-}(\varphi)\right| \leq \sup _{x}\left|\varphi^{(\alpha)}(x)\right| \text { for all } t \geq 0\right)=1 .
$$

To prove (4.8) we proceed as follows. By (3.16) we can find 
for each $\rho>0$ an $A<\infty$ such that

$$
\sup _{\alpha \geq 1} P^{(\alpha)}\left(\sup _{0 \leq t \leq T}\left(\left|N_{t}\left(\left|\nabla h_{\beta}\right|^{2}\right)\right|+\left|N_{t}\left(1 / 2 \Delta h_{\beta}\right)\right|+\left|N_{t}\left(h_{\beta}^{2}\right)\right|\right) \geq A\right)<\rho .
$$

Thus if

$$
\tau=\inf \left\{t \geq 0:\left|N_{t}\left(\left|\nabla h_{\beta}\right|^{2}\right)\right|+\left|N_{t}\left(1 / 2 \Delta h_{\beta}\right)\right|+\left|N_{t}\left(h_{\beta}^{2}\right)\right| \geq A\right\},
$$

then $P^{(\alpha)}(\tau \leq T)<\rho$ for all $\alpha \geq 1$. Now define

$$
X(t)=N_{t \wedge \tau}\left(h_{\beta}\right)-\int_{0}^{t \wedge \tau} N_{s}\left(1 / 2 \Delta h_{\beta}\right) d s
$$

Clearly, since $\rho$ was arbitrary, (4.8) will follow once we show that

$$
\lim _{\delta \rightarrow 0} \sup _{\alpha \geq 1} P^{(\alpha)}\left(\tilde{\omega}_{X}(\delta) \geq \varepsilon / 4\right)=0 .
$$

Using Censov's criterion (cf. Theorem 15.6 in [1]), we will have (4.9) if we can prove that

$$
\sup _{\alpha \geq 1} E^{P^{(\alpha)}}\left[\left(X\left(t_{3}\right)-X\left(t_{2}\right)\right)^{2}\left(X\left(t_{2}\right)-X\left(t_{1}\right)^{2}\right] \leq B^{2}\left(t_{3}-t_{1}\right)^{2}\right.
$$

for some $B<\infty$ and all $0 \leq t_{1}<t_{2}<t_{3} \leq T$. But we saw in section (3) that $\left(X(t), \mathscr{M}_{t}, P^{(\alpha)}\right)$ is a martingale; and, by (3.19), it is clear that

$$
\left(X^{2}(t)-B t, \mathscr{M}_{t}, P^{(\alpha)}\right)
$$

is a supermartingale when $B=A+1+\|\| \Delta h_{\beta} \|^{2}$. Thus

$$
\begin{aligned}
& E^{P^{(\alpha)}}\left[X\left(X\left(t_{3}\right)-X\left(t_{2}\right)\right)^{2}\left(X\left(t_{2}\right)-X\left(t_{1}\right)\right)^{2}\right] \\
& \quad=E^{P^{(\alpha)}}\left[\left(E\left[X^{2}\left(t_{3}\right) \mid \mathscr{M}_{t_{2}}\right]-\left(X\left(t_{2}\right)\right)^{2}\right)\left(X\left(t_{2}\right)-X\left(t_{1}\right)\right)^{2}\right] \\
& \quad \leq B\left(t_{3}-t_{2}\right) E^{P^{(\alpha)}}\left[\left(X\left(t_{2}\right)-X\left(t_{1}\right)\right)^{2}\right] \\
& \quad \leq B^{2}\left(t_{3}-t_{2}\right)\left(t_{2}-t_{1}\right) \leq \mathrm{B}^{2}\left(t_{3}-t_{1}\right)^{2} .
\end{aligned}
$$

Q. E. D.

We have now proved the next theorem (the details here, given (4.4) and (4.5), are essentially the same as those given in preparation for Theorem 1.23)

(4.10) Theorem: The family $\left\{P^{(\alpha)}: \alpha \geq 1\right\}$ is precompact on $D\left([0, \infty), \mathscr{H}_{d+3}\right)$. Moreover, if $\alpha_{n} \rightarrow \infty$ and $P=\lim P^{\left(\alpha_{n}\right)}$ then $P$ is concentrated on $C\left([0, \infty), \mathscr{H}_{d+3}\right)$ and therefore on $\stackrel{n \rightarrow \infty}{C}\left([0, \infty), \mathscr{S}^{\prime}\left(R^{d}\right)\right)$. 
We are now ready to prove the main result of this section.

(4.11) Theorem: If $d \geq 3$ then $P^{(\alpha)} \rightarrow P$ as $\alpha \rightarrow \infty$ where $P$ is the Ornstein-Uhlenbeck process with characteristics $1 / 24$ and I starting from 0.

Proof: Because of Theorem (4.10) and Theorem (1.4), it suffices to show that if $\alpha_{n} \rightarrow \infty$ and $P^{\left(\alpha_{n}\right)} \rightarrow Q$, then $Q\left(N_{0}=0\right)=1$, and for all $\varphi \in \mathscr{S}\left(R^{d}\right)$

$$
\sup _{0 \leq t \leq T} E^{Q}\left[\left|N_{s}(\varphi)\right|\right]<\infty, \quad T>0
$$

and

$$
f\left(N_{t}(\varphi)\right)-\int_{0}^{t} N_{s}(1 / 2 \Delta \varphi) f^{\prime}\left(N_{s}(\varphi)\right) d s-\frac{\|\varphi\|^{2}}{2} \int_{0}^{t} f^{\prime \prime}\left(N_{s}(\varphi)\right) d s
$$

is a $Q$-martingaie whenever $f \in C_{0}^{\infty}\left(R^{1}\right)$.

We first note that $Q\left(N_{0}=0\right)=1$ and that

$$
\sup _{0 \leq t \leq T} E^{Q}\left[\left|N_{s}(\varphi)\right|\right]<\infty, \quad T>0 \text { and } \varphi \in \mathscr{S}\left(R^{d}\right) .
$$

Indeed, each of these facts is an immediate consequence of (3.14).

Now let $\varphi \in \mathscr{S}\left(R^{d}\right), f \in C_{0}^{\infty}\left(R^{1}\right)$ and $0 \leq t_{1}<t_{2}$ be given and suppose that $\Phi: D\left([0, \infty), \mathscr{H}_{d+3}\right) \rightarrow[0,1]$ is a continuous $\mathscr{M}_{t}$-measurable function. We must show that

$$
\begin{gathered}
E^{Q}\left[\left(f\left(N_{t_{2}}(\varphi)\right)-f\left(N_{t_{1}}(\varphi)\right)-\int_{t_{1}}^{t_{2}} N_{s}(1 / 2 \Delta \varphi) f^{\prime}\left(N_{s}(\varphi)\right) d s\right.\right. \\
\left.\left.-\frac{\|\varphi\|^{2}}{2} \int_{t_{1}}^{t_{2}} f^{\prime \prime}\left(N_{s}(\varphi)\right) d s\right) \Phi\right]=0 .
\end{gathered}
$$

Let

$$
\bar{N}_{t}^{(\alpha)}(\psi)=N_{t}(\psi)+\int \psi^{(\alpha)}(x) d x, \quad \psi \in \mathscr{S}\left(R^{d}\right) .
$$

Then, by (3.15) :

$$
\begin{aligned}
& E^{P^{(\alpha)}}\left[\left(f\left(N_{t_{1}}(\varphi)\right)-f\left(N_{t_{2}}(\varphi)\right)-\int_{t_{1}}^{t_{t_{2}}} N_{s}(1 / 2 \Delta \varphi) f^{\prime}\left(N_{s}(\varphi)\right) d s\right) \Phi\right] \\
& =E^{p(\alpha)}\left[\left(\alpha^{2} \int_{t_{1}}^{t_{2}} \bar{N}_{s}^{(\alpha)}\left(K_{\varphi(\alpha)(\cdot)} f\left(\bar{N}_{s}(\varphi)\right)\right) d s\right) \Phi\right]
\end{aligned}
$$




$$
+\frac{\alpha^{-(d+2) / 2}}{2} E^{P^{(\alpha)}}\left[\left(\int_{t_{1}}^{t_{2}} \bar{N}_{s}^{(\alpha)}\left(|\nabla \varphi|^{2}\right) f^{\prime \prime}\left(N_{s}(\varphi)\right) d s\right) \Phi\right]
$$

where

$$
K_{a} f(x) \equiv \frac{f(x+a)+f(x-a)}{2}-f(x), \quad a \in R^{1}
$$

Since $\sup _{\alpha \geq 1} E^{P^{(\alpha)}}\left[\sup _{0 \leq t \leq T}\left(N_{t}(\phi)\right)^{2}\right]<\infty$ for all $T>0$ and $\phi \in \mathscr{S}\left(R^{d}\right)$, it is clear from the preceding that all we need do is show that (in the notation of section (3)) :

$$
\begin{gathered}
\lim _{\alpha \rightarrow \infty} E^{\tilde{P}}\left[\alpha^{2} \mid \int_{t_{1}}^{t_{2}}\left(\tilde{\eta}_{\alpha^{2} s}\left(K_{\varphi(\alpha)} f\left(\eta_{s}^{(\alpha)}(\varphi)\right)\right)\right.\right. \\
\left.\left.-\frac{\|\left.\varphi\right|^{2}}{2 \alpha^{2}} f^{\prime \prime}\left(\eta_{s}^{(\alpha)}(\varphi)\right)\right) \mathrm{d} s \mid\right]=0 .
\end{gathered}
$$

But, since $f \in C_{0}^{\infty}\left(R^{d}\right)$, there is an $A<\infty$ such that:

$$
\begin{aligned}
\mid K_{\varphi^{(\alpha)}(y)} f(x) & -\left.\frac{1}{2} f^{\prime \prime}(x)\left(\varphi^{(\alpha)}(y)\right)^{2}|\leq A| \varphi^{(\alpha)}(y)\right|^{3} \\
& \leq \frac{\bar{A}}{\alpha^{(d+2) / 2}}\left(\varphi^{(\alpha)}(y)\right)^{2} .
\end{aligned}
$$

where $\bar{A}=A \sup _{x}|\varphi(x)|$. Thus

$$
\begin{aligned}
\alpha^{2} E^{\tilde{P}} & {\left[\left|\tilde{\eta}_{\alpha^{2}{ }_{s}}\left(K_{\varphi^{(\alpha)}(\cdot)} f\left(\eta_{s}^{(\alpha)}(\varphi)\right)-\frac{1}{2} f^{\prime \prime}\left(\eta_{s}^{(\alpha)}(\varphi)\right)\left(\varphi^{(\alpha)}(\cdot)\right)^{2}\right)\right|\right] } \\
& \leq \frac{\bar{A} \alpha^{2}}{\alpha^{(d+2) / 2} E^{p}\left[\tilde{\eta}_{\alpha^{2} s}\left(\left(\varphi^{(\alpha)}\right)^{2}\right)\right]} \\
& =\frac{\bar{A} \alpha^{d+2}}{\alpha^{(3 d+6) / 2}} \int \varphi^{2}(x) d x \rightarrow 0
\end{aligned}
$$

at a rate which is independent of $s$. Thus we need only show that

$$
\alpha^{2} E^{\vec{P}}\left[\left|\frac{1}{2} f^{\prime \prime}\left(\eta_{s}^{(\alpha)}(\varphi)\right) \tilde{\eta}_{\alpha^{2} s}\left(\left(\varphi^{(\alpha)}\right)^{2}\right)-\frac{\|\varphi\|^{2}}{2 \alpha^{2}} f^{\prime \prime}\left(\eta_{s}^{(\alpha)}(\varphi)\right)\right|\right] \rightarrow 0,
$$

as $\alpha \rightarrow \infty$, uniformly on bounded $s$-intervals. But to do this reduces to proving

$$
\alpha^{2} E^{\tilde{P}}\left[\left|\tilde{\eta}_{\alpha^{2} s}\left(\left(\varphi^{(\alpha)}\right)^{2}\right)-\frac{\|\varphi\|^{2}}{\alpha^{2}}\right|\right] \rightarrow 0
$$

uniformly on bounded $s$-intervals. To this end, note that 


$$
\left(\varphi^{(\alpha)}\right)^{2}=\frac{1}{\alpha^{(d+2) / 2}}\left(\varphi^{2}\right)^{(\alpha)}
$$

and

$$
\frac{\|\varphi\|^{2}}{\alpha^{2}}=\frac{1}{\alpha^{(d+2) / 2}} \int\left(\varphi^{2}\right)^{(\alpha)}(x) d x .
$$

Hence

$$
\alpha^{2}\left(\tilde{\eta}_{\alpha^{2} s}\left(\left(\varphi^{(\alpha)}\right)^{2}\right)-\frac{\|\varphi\|^{2}}{\alpha^{2}}\right)=\frac{1}{\alpha^{(d-2) / 2}} \eta_{s}^{(\alpha)}(\varphi),
$$

and so, by (3.14):

$$
\begin{gathered}
\alpha^{2} E^{\tilde{P}}\left[\left|\tilde{\eta}_{\alpha^{2} s}\left(\left(\varphi^{(\alpha)}\right)^{2}\right)-\frac{\|\varphi\|^{2}}{\alpha^{2}}\right|\right] \\
\quad \leq \frac{1}{\alpha^{(d-2) / 2}} E^{\tilde{P}}\left[\left(\eta_{s}^{(\alpha)}(\varphi)\right)^{2}\right]^{1 / 2} \\
\quad \leq \frac{1}{\alpha^{(d-2) / 2}}(C+s)^{1 / 2}\|\varphi\| .
\end{gathered}
$$

We have therefore proved (4.13).

Q. E. D.

The reader should remark that it is only in the derivation of (4.13) that we have used the assumption that $d \geq 3$. When $d=2$ one can proceed as follows. Denote by $\hat{P}^{(\alpha)}, \alpha \geq 1$, the distribution under $\tilde{P}$ of $\tilde{\eta}_{\alpha^{2} s}\left(\varphi^{(\alpha)}\right)$. Observe that $\hat{P}^{(\alpha)}$ is concentrated on paths with values in the space of "tempered measures" (i. e. non-negative elements of $\left.\mathscr{S}^{\prime}\left(R^{2}\right)\right)$. One can then use the preceding to show that $\left\{\hat{P}_{\alpha}: \alpha \geq 1\right\}$ is precompact and that any limit $\hat{P}$ as $\alpha \rightarrow \infty$ has the properties that

$$
\hat{P}\left(N_{0}=\text { Lebesgue measure }\right)=1
$$

and

$$
\text { (4. 14) } f\left(N_{t}(\varphi)\right)-\int_{0}^{t} N_{s}\left(\frac{1}{2} \Delta \varphi\right) f^{\prime}\left(N_{s}(\varphi)\right) d s-\frac{1}{2} \int_{0}^{t} N_{s}\left(\varphi^{2}\right) f^{\prime \prime}\left(N_{s}(\varphi)\right) d s
$$

is a $\hat{P}$-martingale for all $\varphi \in \mathscr{S}\left(R^{2}\right)$ and $f \in C_{0}^{\infty}\left(R^{1}\right)$. G. Papanicolau has pointed out to us that if $\left\{\hat{P}_{N}: N\right.$ a tempered measure $\}$ is a family of measures satisfying $\hat{P}_{N}\left(N_{0}=N\right)=1$ and (4.14) is a $\hat{P}_{N}$ martingale for all $\varphi \in \mathscr{S}\left(R^{2}\right)$ and $f \in C_{0}^{\infty}\left(R^{2}\right)$ then it is the Markov family of 
measures studied by Dawson in [2], [13], and [14]. To see this let $g(t, x)$ solve the equation $\frac{\partial g}{\partial t}=\frac{1}{2} \Delta g-\frac{1}{2} g^{2}$ with $g(0, x)=\varphi(x) \geq 0$. Then setting $f(x)=e^{-x}$ in (4.14) one sees that for all tempered measures $N, \exp \left[-N_{t}(g(T-t, \bullet))\right]$ is a $\hat{P}_{N}$ martingale for $0 \leq t \leq T$. (A careful proof of this uses critically the positivity of $\varphi$ and hence of $g(t, \cdot)$ and $\left.N_{t}(g(T-t, \cdot))\right)$. Thus

$$
E^{P_{N}}\left[e^{-N(\varphi)}\right]=e^{-N(g(T, \cdot))} .
$$

This proves uniqueness of the solution to the martingale problem in (4. 14) and identifies the process.

Dawson [14] has shown that in 2 dimensions

$$
\lim _{t-\infty} \hat{P}^{L e b}\left(\left|N_{t}(\varphi)\right|>\varepsilon\right)=0
$$

for all $\varepsilon>0$ and all $\varphi$ with compact support. This corresponds to the situation when $d=2$ mentioned in the first paragraph of the introduction.

If $d=1$, the $\hat{P}^{(\alpha)}$ 's are again tight. Moreover, $E^{\hat{\beta}^{(\alpha)}}\left[\eta_{t}(\varphi)\right]=\frac{1}{\alpha^{1 / 2}} \int \varphi$, and so any limit of the $\hat{P}^{(\alpha)}$ 's must be concentrated at 0 . Thus $\hat{P}^{(\alpha)} \rightarrow \delta_{0}$ as $\alpha \uparrow \infty$.

\section{§5. Ergodic Theorems for Generalized Ornstein- Uhlenbeck Processses}

In this section we consider $O-U$ processes with characteristics $A$ and $I$. Throughout this section we assume that the semi-group, $T_{t}$ generated by $A$ satisfies, in addition to the assumptions in Section 1, the following:

(5.1) $\varphi \rightarrow \int_{0}^{\infty}\left\|T_{t} \varphi\right\|^{2} d t$ is a continuous function on $\mathscr{S}\left(R^{d}\right)$.

Note that if there is a distribution $R \in \mathscr{S}^{\prime}\left(R^{d}\right)$ and

$$
\int_{0}^{\infty} T_{t} \varphi(x) d t=R * \varphi(x),
$$

then (5.1) is satisfied. If $A=\frac{1}{2} \Delta$ and $d \geq 3$ then (5.2) holds with $R(x)=$ constant $/|x|^{d-2}$. If $A=-\Delta^{2}$ and $d \geq 5$ then (5.2) holds with 
$R(x)=$ constant $/|x|^{d-4}$. If $A=\Delta-|x|^{2}$ then

$$
\int_{0}^{\infty}\left\|T_{t} \varphi\right\|^{2} d t=\frac{1}{2} \sum_{\beta}(2|\beta|+d)^{-1}\left(\left(h_{\beta}, \varphi\right)\right)^{2}
$$

and so (5.1) holds (but not (5.2) since $\Delta-|x|^{2}$ is not translation invariant.)

We rely heavily on the following fact:

(5.3) Levy Continuity Theorem (see [9]): If $\left\{C_{n}(\cdot) ; n \geq 1\right\}$ are characteristic functions of measures $\left\{\mu_{n} ; n \geq 1\right\}$ on $\mathscr{S}^{\prime}\left(R^{d}\right)$ and for each $\varphi \in \mathscr{S}\left(R^{d}\right) C_{n}(\varphi) \rightarrow C(\varphi)$ where $C(\bullet)$ is continuous, then $C$ is the characteristic function of a measure $\mu$ and $\mu_{n}$ converges weakly to $\mu$.

(5.4) Lemma: Let $\left\{P_{M}: M \in \mathscr{S}^{\prime}\left(R^{d}\right)\right\}$ be the $O-U$ process with characteristics $A$ and $I$. Then there is a probability measure $\mu$ on $\mathscr{S}^{\prime}\left(R^{d}\right)$ with characteristic function

$$
C(\varphi)=\exp \left[-\frac{1}{2} \int_{0}^{\infty}\left\|T_{s} \varphi\right\|^{2} d s\right]
$$

and $\mu$ satisfies

$$
\int P_{M}\left(N_{t} \in \Gamma\right) \mu(d M)=\mu(\Gamma) \text { for all measurable } \Gamma \subseteq \mathscr{S}^{\prime}\left(R^{d}\right) .
$$

(i. e., $\mu$ is stationary for $\left.\left\{P_{M}: M \in \mathscr{S}^{\prime}\left(R^{d}\right)\right\}\right)$.

Proof: From Theorem (1.4) (with $M=0$ ) we see that for all $t>0$

$$
\exp \left[-\frac{1}{2} \int_{0}^{t}\left\|T_{s} \varphi\right\|^{2} d s\right]
$$

is the characteristic function of a measure. Hence the existence of $\mu$ with characteristic function $C$ follows from (5.1) and (5.3). To check (5.5) it suffices to show that

$$
\int E^{P_{M}}\left[e^{i N_{t}(\varphi)}\right] \mu(d M)=C(\varphi) .
$$

But this follows immediately from Theorem (1.4).

Q. E. D.

Now if $\mu \in \mathscr{S}^{\prime}\left(R^{d}\right)$ we define $\mu_{M}$ to be $\mu$ shifted by $M$. Then 
$\mu_{M}$ has characteristic function

$$
C_{M}(\varphi)=\exp \left[i M(\varphi)-\frac{1}{2} \int_{0}^{\infty}\left\|T_{s} \varphi\right\|^{2} d s\right]
$$

Our next theorem shows that all stationary measures of the $O-U$ process with characteristics $A$ and $I$ must be a special kind of average of the $\mu_{M}$ 's.

(5.7) Theorem: Let $\nu$ be a stationary measure for the $O-U$ process with characteristics $A$ and $I$. Then

$$
\nu=\int \mu_{M} m(d M)
$$

where $m$ satisfies

$$
m(N \in \Gamma)=m\left(T_{t} N \in \Gamma\right)
$$

for all $t \geq 0$ and measurable $\Gamma \subseteq \mathscr{S}^{\prime}\left(R^{d}\right)$.

Conversely any such measure $m$ defines a stationary measure $\nu$ by (5. 8).

Proof: Suppose $\nu$ is stationary. Then for all $t \geq 0$

$$
\begin{aligned}
\int e^{i N(\varphi)} \nu(d N) & =\int E^{P_{M}}\left[e^{i N t(\varphi)}\right] \nu(d M) \\
& =\int e^{i M\left(T_{t} \varphi\right)-\frac{1}{2} \int_{0}^{t_{0}|| T_{s} \|^{2} d s} \nu(d M) .}
\end{aligned}
$$

Hence $\int e^{i \mu\left(T_{t}^{\varphi)}\right.} \nu(d \mu)$, which is the characteristic function of a measure $\nu_{t}$, is equal to $e^{\frac{1}{2} \int_{0}^{t}\left\|T s_{s}\right\|^{2} d s} \int e^{i N(\varphi)} \nu(d N)$. As $t$ goes to infinity

$$
\int e^{i N(\varphi)} \nu_{t}(d N) \rightarrow e^{\frac{1}{2} \int_{0}^{\infty} \| T \cdot e^{\mid \| d s}} \int e^{i N(\varphi)} \nu(d N)
$$

which is continuous by (5.1); and hence by (5.3) is the characteristic function of a measure $m$. Thus

$$
\begin{aligned}
\int e^{i N(\varphi)} \nu(d N) & =\int e^{i M(\varphi)-\frac{1}{2} \int_{0}^{\infty} \| T t^{\varphi \mid \|^{2} d t} m(d M)} \\
& =\iint e^{i N(\varphi)} \mu_{M}(d N) m(d M)
\end{aligned}
$$


and therefore $\nu=\int \mu_{M} m(d M)$. Also

$$
\begin{aligned}
\int e^{i N(T, \varphi)} m(d N) & =\lim _{s \rightarrow \infty} \int e^{i N\left(T_{t} \varphi\right)} \nu_{s}(d N) \\
& =\lim _{s \rightarrow \infty} \int e^{i N\left(T_{s} T_{t}^{\varphi} \varphi\right)} \nu(d N)=\int e^{i N(\varphi)} m(d N),
\end{aligned}
$$

which proves (5.9).

Conversely suppose (5.9) holds and define $\nu$ by (5.8). Then

$$
\begin{aligned}
\int E^{P_{M}}\left[e^{i N} t^{(\varphi)}\right] \nu(d M) & =\int m(d M) \int e^{i N(T, \varphi)-\frac{1}{2} \int_{0}^{t}\left\|T_{s}\right\|^{2} \|^{2} d s} \mu_{M}(d N) \\
& =\int e^{i M\left(T t^{\varphi)}-\frac{1}{2} \int_{0}^{\infty}\left\|T_{s} T_{t}\right\|^{2}\left\|^{2} d s-\frac{1}{2} \int_{0}^{t}\right\| T_{s} \varphi \|^{2} d s\right.} m(d M) \\
& =\int e^{i M(\varphi)} \nu(d M)
\end{aligned}
$$

and so $\nu$ is stationary.

Q. E. D.

The next few lemmas are concerned with measures satisfying (5. 9).

(5.12) Lemma: If for all $\varphi \in \mathscr{S}\left(R^{d}\right), T_{t}(\varphi) \rightarrow 0$ in $\mathscr{S}\left(R^{d}\right)$ as $t \rightarrow \infty$ then the only measure satisfying (5.9) is the one concentrated on zero. In this case, for all starting points $M, P_{M} \circ N_{t}^{-1}$ converges weakly to $\mu$ as $t$ goes to infinity.

Proof: The first part of this lemma follows from (5.3) and the second part follows from (5.3) and Theorem (4.1). $\quad$ Q. E. D.

(5.13) Example: If $A=\Delta-|x|^{2}$ then the hypotheses of Lemma (5.12) are satisfied (see (A. 10) and (A. 15)).

(5.14) Lemma: If $m$ satisfies (5.9) and is such that $m\left(\left\{N\right.\right.$ : there is an $n$ such that $\left.\left.A^{n} N=0\right\}\right)=1$ then $m\left(\left\{N: T_{t} N=N\right.\right.$ for all $\left.\left.t\right\}\right)=1$.

Proof: Using induction on (1.1) one can easily show that for 
all $N \in \mathscr{S}^{\prime}\left(R^{d}\right)$ and all $\varphi \in \mathscr{S}\left(R^{d}\right)$

$$
N\left(T_{t} \varphi\right)-N(\varphi)=\sum_{k=1}^{n-1} \frac{t^{k}}{k !} N\left(A^{k} \varphi\right)+\frac{1}{(n-1) !} \int_{0}^{t}(t-s)^{n-1} N\left(A^{n} T_{s} \varphi\right) d s .
$$

Thus if $N$ is such that $A^{n} N=0$ for some $n$, then $N\left(T_{t} \varphi\right)-N(\varphi)$ as a function of $t$ is a polynomial with zero constant term. In particular if $N\left(T_{t} \varphi\right) \neq N(\varphi)$ for some $t$ then $\left|N\left(T_{t} \varphi\right)-N(\varphi)\right|$ goes to infinity as $t$ goes to infinity. From this observation it is an easy exercise to show that since $N\left(T_{t} \varphi\right)$ and $N(\varphi)$ have the same law under $m$ for all $t$, $N\left(T_{t} \varphi\right)$ must equal $N(\varphi)$ with $m$ probability one. Since $T_{t} \varphi$ is continuous in $t$ and $\varphi$ separately and $[0, \infty)$ and $\mathscr{S}\left(R^{d}\right)$ are separable, it follows that

$$
m\left(N=T_{t} N \text { for all } t \geq 0\right)=1 .
$$

Q. E. D.

We want to show that the hypotheses of Lemma (5.14) are satisfied by $A=\frac{1}{2} \Delta$, but first we need the following result about tempered distributions.

(5.15) Lemma : Let $\sigma \in C^{\infty}\left(R^{d}\right)$ and assume that $\sigma$ and its derivatives grow no faster than polynomials. Let $N$ be a tempered distribution with support $S$ and assume that $S \subset\{x: \sigma(x)=0\}$. Then there is an integer $m$ such that $\sigma^{m} N=0$.

Proof: Suppose first that $S$ is compact. Then $N$ is a distribution with compact support and hence there is a number $C>0$ and an integer $n$ such that for all $\varphi \in \mathscr{S}\left(R^{d}\right)$

$$
|N(\varphi)| \leq C \sum_{|\alpha| \leq n} \sup _{y}\left|D^{\alpha} \varphi(y)\right| \text {. }
$$

We take $m=n+1$ and show that $\sigma^{m} N=0$.

Let $\rho \in C_{0}^{\infty}\left(R^{d}\right)$ with support in $\{x:|x| \leq 1\}, \rho \geq 0$ and $\int \rho(x) d x=1$. Define $\rho_{\varepsilon}(x)=\varepsilon^{-d} \rho\left(\frac{x}{\varepsilon}\right)$ and $\psi_{\mathrm{c}}(x)=\rho_{\varepsilon} * I_{s^{2 \varepsilon}}$, where $S^{\varepsilon}=\{x: \operatorname{dist}(x, S) \leq \varepsilon\}$. Note that $\psi_{\mathrm{s}}(x)=1$ if $x \in S^{\mathrm{s}}$ and thus, since supp $\sigma^{m} N \subset S, \phi_{\varepsilon} \sigma^{m} N=\sigma^{m} N$. Next note that $\left|D^{\alpha} \psi_{\mathrm{s}}(y)\right| \leq \varepsilon^{-|\alpha|} \int\left|D^{\alpha} \rho(x)\right| d x$ and that $\sup _{x \in S^{3 \varepsilon}}\left|\sigma^{j}(x)\right|$ is bounded by a constant times $\varepsilon^{j}$. Thus if $0<\varepsilon \leq 1$ and $|\alpha|<m$, then 
$\sup _{x \in S^{3 \varepsilon}}\left|D^{\alpha} \sigma^{m}(x)\right|$ is bounded by a constant times $\varepsilon^{m-|\alpha|}$. Since $\psi_{\varepsilon}(x)=0$ if $x \notin \mathrm{S}^{3 e}$ it follows easily that $\sup _{y}\left|D^{\alpha} \psi_{c} \sigma^{m}(y)\right|$ is bounded by a constant times $\varepsilon^{m-|a|}$. Thus there is a constant $\bar{C}$ such that for all $0<\varepsilon \leq 1$

$$
\begin{aligned}
\left|\sigma^{m} N(\varphi)\right| & =\left|N\left(\psi_{\mathrm{e}} \sigma^{m} \varphi\right)\right| \leq C \sum_{|\alpha| \leq s} \sup _{y}\left|D^{\alpha} \psi_{\mathrm{a}} \sigma^{m} \varphi\right| \\
& \leq \varepsilon \bar{C} \sum_{|\alpha| \leq n} \sup _{y}\left|D^{\alpha} \varphi\right| ;
\end{aligned}
$$

that is, $\sigma^{m} N=0$.

If $S$ is not compact let $\gamma_{n} \in C_{0}^{\infty}$ be such that

$$
\gamma_{n}(x)=\left\{\begin{array}{lll}
1 & \text { if } & |x| \leq n \\
0 & \text { if } & |x| \geq n+1
\end{array}\right.
$$

Then $\gamma_{n} N$ has compact support and satisfies the hypotheses of the lemma. Thus by the above there is an $m$, which depends only on the order of $N$, such that $\gamma_{n} \sigma^{m} N=0$. (Here we rely on the assumption that $N$ is tempered.) In particular the support of $\sigma^{m} N$ does not intersect $\{x:|x| \leq n\}$. Since this is true for all $n, \sigma^{m} N=0$.

Q. E. D.

We want to apply the above lemma to the following situation. Suppose $\sigma \in C^{\infty}\left(R^{d}\right), \sigma \leq 0, \sigma(x)=\sigma(-x)$, and $\sigma$ and its derivatives grow no faster than polynomials. If $\varphi \in \mathscr{S}_{c}\left(R^{d}\right)$ let $\hat{\varphi}$ denote its Fourier transform. Define an operator $A$ on $\mathscr{S}_{C}\left(R^{d}\right)$ by $\widehat{A \varphi}=\sigma \hat{\varphi}$. Since $\sigma \leq 0 A$ is non-positive definite; and, since $\sigma(x)=\sigma(-x), A \varphi$ is real valued if $\varphi$ is real valued. $A$ generates a self-adjoint semigroup, $T_{t}$, given by $\widehat{T_{t} \varphi}=e^{t o} \hat{\varphi}$.

If $N$ is a tempered distribution $\hat{N}(\varphi) \equiv N(\hat{\varphi})$.

(5. 17) Lemma: Let $A$ and $T_{t}$ be as above and let $m$ be a probability measure on $\mathscr{S}^{\prime}\left(R^{d}\right)$ satisfying (5.9). Then

$$
m\left(T_{t} N=N \text { for all } t\right)=1 .
$$

Proof: By Lemma (5.14) it suffices to show that

$$
m\left(\left\{N: \text { there is an } m \text { such that } A^{m} N=0\right\}\right)=1 .
$$


But $\widehat{A^{m} N}=\sigma^{m} \hat{N}$, and thus by Lemma (5.15), (5.18) will follow if we can show that

$$
m(\{N: \operatorname{supp} \hat{N} \subseteq \text { zeros of } \sigma\})=1 .
$$

To see (5.19) let $\varphi \in \mathscr{S}_{c}\left(R^{d}\right)$ be such that support $\varphi \cap\{x: \sigma(x)=0\}=\phi$. Then as $t$ goes to infinity $e^{\sigma t} \varphi$ converges to 0 in $\mathscr{S}_{C}\left(R^{d}\right)$. Now since for all $t \geq 0$ the laws under $m$ of $N$ and $T_{t} N$ are the same, it follows that the laws of $\hat{N}$ and $\widehat{T_{t} N}$ are the same. Hence $\hat{N}(\varphi)$ and $\widehat{T_{t} N}(\varphi)=\hat{N}\left(e^{t \sigma} \varphi\right)$ have the same law for all $t \geq 0$. But $\hat{N}\left(e^{t \sigma} \varphi\right) \rightarrow 0$ as $t \rightarrow \infty$ for all $N \in \mathscr{S}^{\prime}\left(R^{d}\right)$. (5.14) follows easily from this.

Q. E. D.

(5. 20) Example: If $A=\frac{1}{2} \Delta$ and $\sigma(x)=-\frac{1}{2}|x|^{2}$, then $A$ and $\sigma$ are related as in Lemma (5.17). Thus it follows from Lemmas (5.17) and (5.7) that all stationary measures for the O-U process with characteristics $\frac{1}{2} \Delta$ and $I$ are convex combinations of $\left\{\mu_{H}: H\right.$ a harmonic function in $\left.\mathscr{S}^{\prime}\left(R^{d}\right)\right\}$. It is also easy to see that the $\mu_{H}$ are the extreme points of the set of stationary measures.

If $\nu$ is a stationary measure given by (5.8) with $m$ satisfying (5.9), then whether or not $\nu$ makes the process time reversible seems to be closely related to whether or not $m$ satisfies

$$
m\left(\left\{N: T_{t} N=N\right\}\right)=1
$$

for all $t \geq 0$. One direction of this relation is made clear by the following lemma.

(5. 22) Lemma: If $\nu$ is given by (5.8) and $m$ satisfies (5.21) then $\nu$ makes the process time reversible, i. e., for all $0 \leq s<t$ and $\varphi, \phi \in$ $\mathscr{S}\left(R^{d}\right)$

$$
\int E^{P_{M}}\left[e^{i\left(N_{s}(\varphi)+N_{t}(\psi)\right)}\right] \nu(d M)=\int E^{P_{M}}\left[e^{i\left(N_{s}(\varphi)+N_{t}(\varphi)\right)}\right] \nu(d M) .
$$

Proof: By making use of Theorem (1.4) it is a straightforward computation to check that 


$$
\begin{aligned}
& \int E^{P_{M}}\left[e^{i\left(N_{\theta}(\varphi)+N_{t}(\psi)\right)}\right] \nu(d M)
\end{aligned}
$$

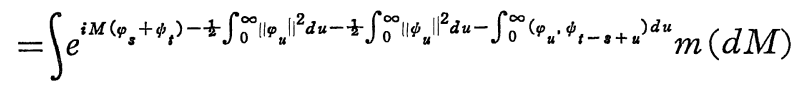

and

$$
\begin{aligned}
& \int E^{P_{M}}\left[e^{i\left(N_{s}(\psi)+N_{t}(\varphi)\right)}\right] \nu(d M) \\
= & \int e^{i M\left(\varphi_{s}+\varphi_{t}\right)-\frac{1}{2} \int_{0}^{\infty}\left\|\varphi_{u}\right\|^{2} d u-\frac{1}{2} \int_{0}^{\infty}\left\|\psi_{u}\right\|^{2} d u-\int_{0}^{\infty}\left(\varphi_{\left.t-s+u^{\prime}, \hat{\psi}_{u}\right) d u}\right.} m(d M) .
\end{aligned}
$$

Since $T_{t}$ is self-adjoint $\int_{0}^{\infty}\left(\varphi_{u}, \phi_{t-s+u}\right) d u=\int_{0}^{\infty}\left(\varphi_{t-s+u}, \phi_{u}\right) d u$. If $m$ satisfies (5.21) then $M\left(\phi_{t}\right)=M(\phi)$ and $M\left(\varphi_{s}\right)=M(\varphi)$ for all $t$ and $s(a . s . m)$. and we have the desired result.

Q. E. D.

(5. 24) Remark: From the proof of Lemma (5.22) we see that the condition on $m$ that is equivalent to $\nu$ being time reversible is that for all $0 \leq s<t$ and all $\varphi, \phi \in \mathscr{S}\left(R^{d}\right)$, the law of $\left(M\left(\varphi_{s}\right), M\left(\phi_{t}\right)\right)$ under $m$ is the same as the law of $\left(M\left(\varphi_{t}\right), M\left(\phi_{s}\right)\right)$ under $m$. Clearly (5.21) implies this, but we are unable to decide if the opposite implication holds.

The final goal of this section is to find a Banach space $\left(X,\|\cdot\|_{x}\right)$ contained (topologically) in $\mathscr{S}^{\prime}\left(R^{d}\right)$ such that: i) $X$ is a dense $\mathrm{F}_{\sigma}$ in $\mathscr{S}^{\prime}\left(R^{d}\right)$; ii) for each $M \in X$ the O.-U. process $P_{M}$ with characteristics $\frac{1}{2} \Delta$ and $I$ starting from $M$ is concentrated on $C([0, \infty), X)$; and iii) for each $M \in X$ and all $\Phi \in C_{b}(X), E^{P^{M}}\left[\Phi\left(N_{t}\right)\right] \rightarrow \int \Phi d \mu_{0}$ as $t \rightarrow \infty$, where $\mu_{0}$ is the probability measure on $\mathscr{S}^{\prime}\left(R^{d}\right)$ with characteristic function

$$
\exp \left[-\frac{1}{2} \int_{0}^{\infty}\left\|\varphi_{s}\right\|^{2} d s\right], \quad \varphi \in \mathscr{S}\left(R^{d}\right)
$$

Observe that if we prove iii), then automatically $\mu_{0}(X)=1$. Moreover, we will have shown that $X$ is a state space for the (Feller continuous) Markov family $\left\{P_{M}: M \in X\right\}$ and that $\left\{P_{M}: M \in X\right\}$ is ergodic with $\mu_{0}$ as its unique stationary measure. (The Feller continuity is irrelevant for these considerations but will follow from our estimates below plus the uniqueness which we have already proved in Theorem 
(1. 4)).

In view of our characterization of the stationary measures for $\left\{P_{M}: M \in \mathscr{S}^{\prime}\left(R^{d}\right)\right\}$, it is clear that what has to be done is find a Banach subspace $X$ of $\mathscr{S}^{\prime}\left(R^{d}\right)$ such that $\gamma_{t} * M \rightarrow 0$ strongly as $t \rightarrow \infty$ for all $M \in X$. At the same time, this subspace must be chosen so that $P_{0}$ is concentrated on $C([0, \infty), X)$ and in addition that $t \rightarrow \gamma_{t} * M$ is strongly continuous into $X$ for all $M \in X$ (this latter condition is needed in order to assure that $P_{M}$ is also concentrated on $C([0, \infty)$, $X)$ ). Finally, $X$ must have the property that the one-dimensional time marginals $P_{0} \circ N_{t}^{-1}$ of $P_{0}$ are tight. Considering all the conditions that must be met, it is somewhat surprising that $X$ is so easy to describe.

Given $\rho>0$ and $m \geq 0$, define $\|\cdot\|_{(\rho, m)}$ on $\mathscr{S}^{\prime}\left(R^{d}\right)$ by

$$
\| N||_{(\rho, m)}^{6}=\sum_{n=1}^{\infty} n^{\rho}\left(\left\||| \gamma_{1 / n} * N-N\left|\left\|_{(m)}^{6}+\right\|\right|\left|\gamma_{n-1} * N\right|\right\|_{(m)}^{6}\right),
$$

and let $X_{(\rho, m)}=\left\{N \in \mathscr{S}^{\prime}\left(R^{d}\right):\|N\|_{(\rho, m)}<\infty\right\}$. (See (A. 19) for the definition cf \|\|$\cdot\|\|_{(m)}$.) It is easy to see that each $X_{(\rho, m)}$ is a dense $F_{\sigma}$ in $\mathscr{S}^{\prime}\left(R^{d}\right)$ (density comes from the inclusion $C_{0}^{\infty}\left(R^{d}\right) \subseteq X_{(\alpha, m)}$ ) and that $\left(X_{(\rho, m)},\|\cdot\|_{(\rho, m)}\right)$ is a Banach space.

(5.25) Lemma: For all $M \in X_{(\rho, m)}, t \rightarrow \gamma_{t} * N$ is strongly continuous on $[0, \infty)$ into $X_{(\rho, m)}$ and $\left\|r_{t} * N\right\|_{(\rho, m)} \rightarrow 0$ as $t \rightarrow \infty$. Moreover, for all $0<\rho<\sigma$ and $m \geq 0$, bounded closed subsets of $X_{(\sigma, m)}$ are compact as subsets of $X_{(\rho, m+1)}$.

Proof: Since $\gamma_{t+s}=\gamma_{t} * \gamma_{s}$, we need only check that $t \rightarrow \gamma_{t} * N$ is continuous from $[0,1)$ into $X_{(\rho, m)}$. To this end, note that by (A. 23):

$$
\sup _{0 \leq t \leq 1}\left\|\gamma_{1 / n} * \gamma_{t} * N-\gamma_{t} * N\left|\left\|\left.\right|_{(m)} \leq C_{m}(1)\left|\left\|\gamma_{1 / n} * N-N \mid\right\|_{(m)}\right.\right.\right.\right.
$$

and

$$
\left.\sup _{0 \leq t \leq 1}\left\|\left|\gamma_{n} * \gamma_{t} * N\right|\right\|\right|_{(m)} \leq C_{m}(1)\left\|\left|\gamma_{n} * N\right|\right\|_{(m)}
$$

Thus, in order to prove that

$$
\lim _{\substack{t \rightarrow s \\ t \geq 0}} \| \gamma_{t} * N-\gamma_{s} * N||_{(\rho, m)}=0
$$

for $N \in X_{(\rho, m)}$ and $s \in[0,1)$, it suffices to show that 


$$
\left.\lim _{\substack{t \rightarrow s \\ t \geq 0}}\left\|\gamma_{t} * N-r_{s} * N\right\|\right|_{(m)}=0
$$

When $s>0$, this is obvious from (A. 24). When $s=0$, we argue as follows. Since $N \in X_{(\rho, m)}$, it is clear that $\gamma_{1 / n} * N \rightarrow N$ in $X_{(\rho, m)}$ as $n \rightarrow \infty$ because $\sum_{1}^{\infty} n^{\rho}|| \gamma_{1 / n} * N-N||_{(m)}<\infty$. Thus if $\varepsilon>0$ and we choose $n_{\varepsilon}$ so that $2 C_{m} / n_{\varepsilon}<\varepsilon$ and $\left\|\left|\gamma_{1 / n} * N-N\right|\right\|_{(m)}<\varepsilon$ for $n \geq n_{\mathrm{s}}$, then, by (A. 24), for all $t \in\left[\frac{1}{n+1}, \frac{1}{n}\right]$ where $n \geq n_{\varepsilon}$ :

$$
\begin{aligned}
\left\|\gamma_{t} * N-N \mid\right\|_{(m)} & \leq\left\|\gamma_{t} * N-\gamma_{1 / n} * N \mid\right\|_{(m)}+\varepsilon \\
& \leq C_{m}\left(\frac{1}{n}-t+(1-n t)\right)+\varepsilon \\
& \leq C_{m}\left(\frac{1}{n(n+1)}+\frac{1}{n+1}\right)+\varepsilon \leq 2 \varepsilon .
\end{aligned}
$$

The proof that $\left\|\gamma_{t} * N\right\|_{(\rho, m)} \rightarrow 0$ as $t \rightarrow \infty$ for $N \in X_{(p, m)}$ is an easy consequence of the definition of $X_{(\rho, m)}$ together with (A. 23). Finally, the assertion that bounded subsets of $X_{(a, m)}$ are compact subsets of $X_{(\rho, m+1)}$ is proved in exactly the same way as one shows that a bounded self-adjoint operator is compact if it has a completely discrete spectrum having zero as its only accumulation point. $\quad$ Q. E. D.

(5.26) Lemma: For all $0<\rho<1 / 2$ and $m>d+6$ the measure $P_{0}$ is concentrated on $C\left([0, \infty), X_{(\rho, m)}\right)$ and

$$
\sup _{t \geq 0} E^{P_{0}}\left[\left\|N_{t}\right\|_{(\rho, m)}\right]<\infty .
$$

Proof: First note that for $0 \leq s<t$

$$
E^{P_{0}}\left[\left(N_{t}(\varphi)-N_{s}(\varphi)\right)^{2}\right] \leq 2(t-s)\|\varphi\|^{2},
$$

and that if $X$ is a normal random variable with mean zero, then $E\left[X^{6}\right]=15\left(E\left[X^{2}\right]\right)^{3}$. Thus

$$
E^{P_{0}}\left[\left(N_{t}(\varphi)-N_{s}(\varphi)\right)^{6}\right] \leq 120(t-s)^{3}\|\varphi\|^{6} .
$$

Applying (5.28) to $\gamma_{n-1} * \varphi$ we have

$$
\begin{aligned}
& E^{P^{0}}\left[\left(N_{t}\left(\gamma_{n-1} * \varphi\right)-N_{s}\left(\gamma_{n-1} * \varphi\right)\right)^{6}\right] \leq 120(t-s)^{3}|| \gamma_{n-1} * \varphi \|^{6} \\
& \quad \leq\left.\left. 120 \int e^{-|\theta|^{2}} d \theta(t-s)^{3}\right|_{i} ^{\mid}\right|_{\left.\right|_{L} ^{\infty}\left(R^{d}\right)} ^{\mid 6} /(n-1)^{3} .
\end{aligned}
$$


Also

$$
\begin{aligned}
E^{P_{0}}\left[\left(N_{t}(\varphi)\right)^{6}\right] & \left.=15\left(\int \frac{1-e^{-t|\theta|^{2}}}{|\theta|^{2}}\right)|\hat{\varphi}(\theta)|^{2} d \theta\right)^{3} \\
& \leq 15\left(\frac{\omega_{d}}{d-2}\|\hat{\varphi}\|_{L^{\infty}\left(R^{d)}\right.}^{2}+\|\varphi\|^{2}\right)^{3}
\end{aligned}
$$

and for $n \geq 2$ :

$$
E^{P_{0}}\left[\left(N_{t}\left(\gamma_{n-1} * \varphi\right)\right)^{6}\right] \leq 15\|\hat{\varphi}\|_{L^{\infty}\left(R^{d}\right)}^{6}\left(\int \frac{e^{-|\theta|^{2}}}{|\theta|^{2}} d \theta\right)^{3} /(n-1)^{3(d-2) / 2} .
$$

Using (A. 11) and (A. 12) together with the above, we get:

$$
E^{P}\left[\sum_{n=1}^{\infty} n^{\rho}||_{\gamma} \gamma_{n-1} * N_{t}-\gamma_{n-1} * N_{s} \|_{(m)}^{6}\right] \leq A_{m, d, p}(t-s)^{3}
$$

and

$$
E^{P_{0}}\left[\sum_{n=1}^{\infty} n^{\rho}\left\|\mid \gamma_{n-1} * N_{t}\right\|_{(m)}^{6}\right] \leq B_{m, d, \rho}
$$

for some $A_{m, d, \rho}$ and $B_{m, d, \rho}$ so long as $m>d+6$ and $0<\rho<\frac{1}{2}$.

Next, from (5.28) we have

$$
E^{P_{0}}\left[\left(N_{t}\left(\gamma_{1 / n} * \varphi-\varphi\right)-N_{s}\left(\gamma_{1 / n} * \varphi-\varphi\right)\right)^{6}\right] \leq 120(t-s)^{3} \frac{1}{n^{6}}\left\|\frac{1}{2} \Delta \varphi\right\|^{6} .
$$

Also, taking $s=0$ :

$$
\begin{aligned}
E^{P_{0}}\left[\left(N_{t}\left(\gamma_{1 / n} * \varphi-\varphi\right)\right)^{6}\right] & \leq 15\left(\int_{0}^{\alpha}\left\|\int_{0}^{1 / n} \gamma_{n+1} *\left(\frac{1}{2} \Delta \varphi\right) d v\right\|^{2} d u\right)^{3} \\
& \leq\left(\frac{1}{n} \int_{0}^{1 / n} d v \int_{0}^{\infty}\left\|\gamma_{u+v^{*}} *\left(\frac{1}{2} \Delta \varphi\right)\right\|^{2} d u\right)^{3} \\
& \leq\left(\frac{1}{4 n^{2}}\|\varphi\|\|\Delta \varphi\|\right)^{3} .
\end{aligned}
$$

Since, by (A. 14), $\left\|\Delta h_{\alpha}\right\|^{2} \leq d C(0,2)(2|\alpha|+d)^{2}$, we can use the preceding to find $C_{m, d, \rho}$ and $D_{n, d, \rho}$ such that

$$
E^{P}\left[\sum_{n=1}^{\infty} n^{\rho} \mid \|\left(\gamma_{1 / n} * N_{t}-N_{t}\right)-\left(\gamma_{1 / n} * N_{s}-N_{s} \mid \|_{(m)}^{6}\right] \leq C_{m, d, \rho}(t-s)^{3}\right.
$$

and

(5. 32) $\quad E^{P_{0}}\left[\sum_{n=1}^{\infty} n^{\rho}\left\|\mid \gamma_{1 / n} * N_{t}-N_{t}\right\|_{(m)}^{6}\right] \leq D_{m, d, \rho}$ 
for all $0<\rho<1 / 2$ and $m>d+6$.

Combining (5.29) and (5.30), we arrive at

$$
E^{P_{0}}\left[\left\|N_{t}-N_{s}\right\|_{(\rho, m)}^{6}\right] \leq\left(A_{m, d, \rho}+C_{m, d, \rho}\right)(t-s)^{3},
$$

which implies that $P_{0}\left(C\left([0, \infty), X_{(\rho, m)}\right)\right)=1$ (since we already know that $t \rightarrow N_{t}(\varphi)$ is continuous for all $\left.\varphi \in \mathscr{S}\left(R^{d}\right)\right)$. Finally, (5.30) and (5.31) yield :

$$
\sup _{t \geq 0} E^{P_{0}}\left[\left\|N_{t}\right\|_{(\rho, m)}^{6}\right]<\infty
$$

Q. E. D.

We now have all the necessary facts to complete our program. Let $m>d+7$ and $0<\rho<1 / 2$ be given. By the preceding we know that $P_{0}\left(C\left([0, \infty), X_{(\rho, m)}\right)\right)=1$. Since for every $M \in X_{(\rho, m)} P_{M}$ coincides with the distribution of $\gamma_{t} * M+N_{t}$ under $P_{0}$, it follows from Lemma (5.25) that $P_{M}\left(C\left([0, \infty), X_{(\rho, m)}\right)\right)=1$ and that for any $\Phi \in C_{b}\left(X_{(\rho, m)}\right)$

$$
\lim _{t \rightarrow \infty}\left|E^{P_{0}}\left[\Phi\left(N_{t}\right)\right]-E^{P_{M}}\left[\Phi\left(N_{t}\right)\right]\right|=0 .
$$

Finally, by Lemmas (5.25) and (5.26) we know that the measures $\left\{P_{0} \circ N_{t}^{-1}: t \geq 0\right\}$ are pre-compact on $X_{(\rho, m)}$ and we have already seen that $P_{0} \circ N_{t}^{-1} \rightarrow \mu_{0}$ on $\mathscr{S}^{\prime}\left(R^{d}\right)$; and therefore $P_{M} \circ N_{t}^{-1} \rightarrow \mu_{0}$ on $X_{(\rho, m)}$ for all $M \in X_{(\rho, m)}$. With these remarks we have now proved our final theorem.

(5. 34) Theorem: If $m>d+7$ and $0<\rho<1 / 2$, then the collection $\left\{P_{M}: M \in X_{(\rho, m)}\right\}$ forms a strong Markov, Feller continuous family on $C\left([0, \infty), X_{(\rho, m)}\right)$ and

$$
\lim _{t \rightarrow \infty} E^{P^{M}}\left[\Phi\left(N_{t}\right)\right]=\int \Phi d \mu_{0}
$$

for all $\Phi \in C_{b}\left(X_{(\rho, m)}\right)$.

\section{Appendix}

The purpose of this appendix is to catalogue some facts about Hermite functions. We do not pretend that these facts are unknown, 
in fact many of them have become part of the standard folklore among experts in distribution theory. All that we want to do is compile them here for the convenience of our readers.

Given $k \geq 0$, define $g_{k}: R^{1} \rightarrow R^{1}$ by

$$
g_{k}(x)=(-1)^{k} e^{x^{2} / 2} D_{x}^{k} e^{-x^{2}} .
$$

An obvious and basic fact is that

$$
\text { (A. 2) } \quad \sum_{0}^{\infty} \frac{\lambda^{k}}{k !} g_{k}(x)=e^{-x^{2} / 2+2 x \lambda-\lambda^{2}}, \quad x \in R^{1} \text { and } \lambda \in C \text {. }
$$

From (A. 2) it is not hard to derive :

$$
x g_{k}(x)=\frac{1}{2} g_{k+1}(x)+k g_{k-1}(x)
$$

and

$$
g_{k}^{\prime}(x)=k g_{k-1}(x)-\frac{1}{2} g_{k+1}(x)
$$

where $g_{-1} \equiv 0$. Combining (A.3) and (A. 4), one can easily arrive at

$$
\left(-D_{x}^{2}+x^{2}\right) g_{k}(x)=(2 k+1) g_{k}(x) .
$$

Starting once again with (A. 2), one sees that

$$
\left(g_{k}, g_{l}\right)=\left\{\begin{array}{l}
0 \text { if } k \neq l \\
\pi^{1 / 2} 2^{k} k ! \text { if } k=l .
\end{array}\right.
$$

Thus if

$$
\text { (A. 7) } \quad h_{k}(x)=\left(\pi^{1 / 2} 2^{k} k !\right)^{-1 / 2} g_{k}(x) \text {, }
$$

then $\left\{h_{k}: k \geq 0\right\}$ forms an orthonormal sequence in $L^{2}\left(R^{1}\right)$. The function $h_{k}$ is called the $k^{t h}$ Hermite function. A well-known fact is that $\left\{h_{k}: k \geq 0\right\}$ is a basis in $L^{2}\left(R^{1}\right)$. From (A. 3), (A. 4) and (A. 5), we obtain:

$$
x h_{k}(x)=\left(\frac{k}{2}\right)^{1 / 2} h_{k-1}(x)+\left(\frac{k+1}{2}\right)^{1 / 2} h_{k+1}(x),
$$

$$
h_{k}^{\prime}(x)=\left(\frac{k}{2}\right)^{1 / 2} h_{k-1}(x)-\frac{(k+1)^{1 / 2}}{2} h_{k+1}(x),
$$


and

(A. 10)

$$
\left(-D_{x}^{2}+x^{2}\right) h_{k}(x)=(2 k+1) h_{k}(x) .
$$

Another consequence of (A. 2) is

(A. 11)

$$
\hat{h}_{k}=i^{k} h_{k}, \quad k \geq 0,
$$

where

$$
\hat{f}(\theta) \equiv \frac{1}{(2 \pi)^{1 \prime^{\prime 2}}} \int f(x) e^{i \theta x} d x .
$$

Thus, by the Fourier inversion formula and (A. 8) :

$$
\begin{aligned}
\left|h_{k}(x)\right| & =\frac{1}{(2 \pi)^{1 / 2}}\left|\int h_{k}(\theta) e^{-i \theta x} d \theta\right| \\
& \leq \frac{1}{(2 \pi)^{1 / 2}}\left[\int_{|\theta| \varsigma_{\varepsilon}}\left|h_{k}(\theta)\right| d \theta+\int_{|\theta| \geq_{\varepsilon}} \frac{1}{|\theta|}\left|\theta h_{k}(\theta)\right| d \theta\right] \\
& \leq \frac{1}{(2 \pi)^{1 / 2}}\left[(2 \varepsilon)^{1 / 2}|| h_{k}\left|+\left(\frac{2}{\varepsilon}\right)^{1 / 2}\right|\left|\theta h_{k}\right| \mid\right] \\
& =\frac{1}{\pi^{1 / 2}}\left[\varepsilon^{1 / 2}+\left(\frac{2 k+1}{\varepsilon}\right)^{1 / 2}\right]
\end{aligned}
$$

for each $\varepsilon>0$. Taking $\varepsilon^{1 / 2}=(2 k+1)^{1 / 4}$, we arrive at :

(A. 12) $\quad\left|h_{k}(x)\right| \leq\left(\frac{2}{\pi}\right)^{1 / 2}(2 k+1)^{1 / 4}, \quad k \geq 0$ and $x \in R^{\prime}$.

Next define for $\alpha \in(\{0,1, \ldots, k, \ldots\})^{d}$ the function $h_{\alpha}: R^{d} \rightarrow R^{d}$ by

$$
h_{\alpha}(x)=h_{\alpha_{1}}\left(x_{1}\right) \ldots h_{\alpha_{d}}\left(x_{d}\right), \quad x \in R^{d} .
$$

Properties of the $h_{\alpha}$ 's are easily read off from the corresponding properties of the $h_{k}$ 's. In particular, from (A. 8) and (A.9), we can use induction to show that for all $\mu, \nu \in(\{0,1, \ldots, k, \ldots\})^{d}$ there is a $C(\mu, \nu)$ such that

$$
\text { (A. 14) } \quad\left\|x^{\mu} D_{x}^{\nu} h_{\alpha}\right\|^{2} \leq C(\mu, \nu)(2|\alpha|+d)^{|\mu|+|\nu|} \text {. }
$$

Also, if $\varphi \in \mathscr{S}\left(R^{d}\right)$, then integration by parts shows that $\left\{\left(\varphi, h_{\alpha}\right)\right.$ : $\alpha \geq 0\}$ is rapidly decreasing (i. e. $|\alpha|^{n}\left|\left(\varphi, h_{\alpha}\right)\right| \rightarrow 0$ as $|\alpha| \rightarrow \infty$ for all $n \geq 0)$. Thus if $\varphi \in \mathscr{S}\left(R^{d}\right)$, then from (A. 14) we have:

$$
\left\|x^{\mu} D_{x}^{\nu}\left(\varphi-\sum_{|\alpha| \leq N}\left(\varphi, h_{\alpha}\right) h_{\alpha}\right)\right\| \leq \sum_{|\alpha|>N}\left|\left(\varphi, h_{\alpha}\right)\right||| x^{\mu} D_{x}^{\nu} h_{\alpha} \| \rightarrow 0
$$


as $N \uparrow \infty$. In other words :

$$
\sum_{|\alpha| \leq N}\left(\varphi, h_{\alpha}\right) h_{\alpha} \stackrel{N \uparrow \infty}{\longrightarrow} \varphi \text { in } \mathscr{S}\left(R^{d}\right)
$$

for all $\varphi \in \mathscr{S}\left(R^{d}\right)$. In particular, we can again use (A. 8) and (A.9) to show the existence of $K(\mu, \nu)$ such that

$$
\text { (A. 16) } \quad \|\left. x^{\mu} D_{x}^{\nu} \varphi\right|^{2} \leq K(\mu, \nu) \sum_{\alpha}(2|\alpha|+d)^{|\mu|+|\nu|}\left(\varphi, h_{\alpha}\right)^{2}
$$

for all $\varphi \in \mathscr{S}\left(R^{d}\right)$. In view of (A. 10) and (A. 16), we come to the important conclusion that for each $m \geq 0$ there exist $0<\lambda_{m}<\Lambda_{m}<\infty$ such that

$$
\text { (A. 17) } \quad \lambda_{m} \mid\|\varphi\|\left\|^{(m)} \leq\left(\sum_{|\mu|+|\nu| \leq m}\left\|x^{\mu} D_{x}^{\nu}\right\|^{2}\right)^{1 / 2} \leq \Lambda_{m}\right\| \varphi \|^{(m)}, \quad \varphi \in \mathscr{S}\left(R^{d}\right),
$$

where

$$
\text { (A. 18) } \quad\left(\||\varphi|||^{(m)}\right)^{2} \mathscr{S}\left(\varphi,\left(-\Delta+|x|^{2}\right)^{m} \varphi\right)=\sum_{a}(2|\alpha|+d)^{m}\left(\varphi, h_{\alpha}\right)^{2} \text {. }
$$

We now want to derive some slightly less standard facts about $\mathscr{S}^{\prime}\left(R^{d}\right)$. Namely, let

$$
\text { (A. 19) } \quad \mathscr{H}_{(m)} \equiv\left\{N \in \mathscr{S}^{\prime}\left(R^{d}\right): \||| N||_{(m)} \equiv \sum_{\alpha}(2|\alpha|+d)^{-m}\left(N\left(H_{\alpha}\right)\right)^{2}<\infty\right\} .
$$

It is obvious that $\mathscr{H}_{(m)}$ is a complete separable Hilbert space. In fact $\mathscr{H}_{(m)}$ can be identified as the dual of the completion $\mathscr{H}^{(m)}$ of $\mathscr{S}\left(R^{d}\right)$ with respect to $i \cdot \|^{(m)}$ (for this identification one uses (A. 15)); and the action of $N \in \mathscr{H}_{(m)}$ on $\mathscr{H}^{(m)}$ is given by:

$$
N(\varphi)=\sum_{\alpha}\left(\varphi, h_{\alpha}\right) N\left(h_{\alpha}\right) .
$$

Of course, if $\varphi \in \mathscr{S}\left(R^{d}\right)$ then $N(\varphi)$ given by (A. 20) coincides with the action of $N$ as an element of $\mathscr{S}^{\prime}\left(R^{d}\right)$ on $\varphi$ (this fact justifies our use of the notation $N(\varphi)$ in (A. 20).) As a consequence of these considerations we see that

$$
\|N \mid\|_{(m)}=\sup _{\varphi \in \mathscr{S}\left(R^{d}\right) \backslash(0)} \frac{|N(\varphi)|}{\|\left.\varphi||\right|^{(m)}} .
$$

We are at last ready to prove the next estimate.

(A. 22) Theorem : Define $\gamma_{t} * N, t>0$ and $N \in \mathscr{S}^{\prime}\left(R^{d}\right)$, by $\gamma_{t} * N(\varphi)=$ $N\left(\gamma_{t} * \varphi\right)$. Then for all $m \geq 0$ and $T>0$ there is a $C_{m}(T)<\infty$ such 
that:

(A. 23) $\quad \sup _{0 \leq t \leq T}\left\|\gamma_{t} * N \mid\right\|_{(m)} \leq C_{m}(T)\|\| N \|_{(m)}, \quad N \in \mathscr{H}_{(m)}$.

Moreover, there is a $C_{m}<\infty$ such that for all $0<s<t \leq 1$ :

(A. 24) $\quad\left\|\left|\gamma_{t} * N-\gamma_{s} * N\|\|_{(m)} \leq C_{m}\left(t-s+\left(1-\frac{s}{t}\right)\right)\|N \mid\| \|_{(m)}, \quad N \in \mathscr{H}_{(m)}\right.\right.$.

Proof: In view of (A. 21), (A. 23) and (A. 24) will follow from (A. 25)

$$
\sup _{0 \leq t \leq T}\left\|\gamma_{t} * \varphi \mid\right\|^{(m)} \leq C_{m}(T)\|\| \varphi \|^{(m)}, \quad \varphi \in \mathscr{S}\left(R^{d}\right) \text {, }
$$

and

(A. 26) $\quad\left\|\gamma_{t} * \varphi-\gamma_{s} * \varphi\right\|^{(m)} \leq C_{m}\left(t-s+\left(1-\frac{s}{t}\right)\right)\|\varphi\|^{(m)}, \quad \varphi \in \mathscr{S}\left(R^{d}\right)$,

respectively.

To prove (A. 25) let $\mu$ and $\nu$ be multi-indices satisfying $|\mu|+$ $|\nu| \leq m$. Then

$$
\begin{aligned}
\left\|x^{\mu} D_{x}^{\nu} \gamma_{t} * \varphi\right\|=\left\|D_{\theta}^{\mu} e^{-t|\theta|^{2} / 2}\left(\theta^{\nu} \hat{\varphi}\right)\right\| & =\left.\sum_{\beta \leq \mu}\left(\begin{array}{c}
\mu \\
\beta
\end{array}\right)\right|_{1} D_{\theta}^{\beta}\left(e^{-t|\theta|^{2} / 2}\right) D_{\theta}^{\mu-\beta}\left(\theta^{\nu} \hat{\varphi}\right) \| \\
& =\sum_{\beta \leq \mu}\left(\begin{array}{l}
\mu \\
\beta
\end{array}\right)\left\|P_{\beta}(t, \theta) D^{\mu-\beta}\left(\theta^{\nu} \hat{\varphi}\right)\right\|
\end{aligned}
$$

where $P_{\beta}(t, \theta)$ is a polynomial in $(t, \theta)$ of degree $\beta$ such that $P_{\beta}(0, \theta)$ $\equiv 0$ if $\beta \neq 0$. Using (A. 18), we see from this that

$$
\left\|x^{\mu} D_{x}^{\nu} \gamma_{t} * \varphi\right\| \leq C_{\mu, \nu}(1+t)^{|\mu+\nu|} \mid\|\hat{\varphi}\|^{\mid(m)}
$$

Since $\|\hat{\varphi}\|\left\|^{(m)}=\right\| \varphi \|^{(m)}$, (A. 25) is now obvious. Applying (A. 18), we arrive at (A. 26).

The proof of (A. 26) is similar:

$$
\begin{aligned}
\left\|x^{\mu} D_{x}^{\nu}\left(\gamma_{t} * \varphi-\gamma_{s} * \varphi\right)\right\| & =\left\|D_{\theta}^{\mu}\left(e^{-\frac{1}{t}|\theta|^{2}}-e^{-\frac{f}{t}|\theta|^{2}}\right)\left(\theta^{\nu} \hat{\varphi}\right)\right\|_{1} \\
& \leq \sum_{\substack{\beta \leq \mu \mu \\
\beta \neq 0}}\left(\begin{array}{l}
\mu \\
\beta
\end{array}\right)\left\|e^{-\frac{1}{t}|\theta|^{2}}\left(P_{\beta}\left(t_{1} \theta\right)-P_{\beta}(s, \theta)\right) D_{\theta}^{\mu-\beta}\left(\theta^{2} \hat{\varphi}\right)\right\| \\
& +\sum_{\substack{\beta \leq \mu \\
\beta \neq 0}}\left(\begin{array}{l}
\mu \\
\beta
\end{array}\right)\left\|\left(e^{-\frac{1}{2}|\theta|^{2}}-e^{-\frac{f}{2}|\theta|^{2}}\right) P_{\beta}(s, \theta) D^{\mu-\beta}\left(\theta^{\nu} \hat{\varphi}\right)\right\| \\
& +\left\|\left(e^{-\frac{1}{2}|\theta|^{2}}-e^{-\frac{1}{2}|\theta|^{2}}\right) D_{\theta}^{\mu}\left(\theta^{\nu} \hat{\varphi}\right)\right\|_{\bullet}
\end{aligned}
$$

The first term is bounded by $C_{m}(t-s)\|\mid \varphi\|^{\left({ }^{(m)}\right.}$. As for the last two 
terms, note that $\left|e^{-f|\theta|^{2}}-e^{-f|\theta|^{2}}\right|$ achieves its maximum at any $\theta_{0}$ such that $s e^{-\frac{1}{2}\left|\theta_{0}\right|^{2}}=t e^{-\frac{1}{2}\left|\theta_{0}\right|^{2}}$. Thus

$$
\left|e^{-\frac{1}{2}|\theta|^{2}}-e^{-\frac{\tau}{t}|\theta|^{2}}\right| \leq\left|\frac{s}{t} e^{-\frac{s}{2}\left|\theta_{0}\right|^{2}}-e^{-\frac{s}{2}\left|\theta_{0}\right|^{2}}\right| \mid \leq 1-\frac{s}{t} .
$$

Hence the last two terms are bounded by

$$
C\left(1-\frac{s}{t}\right) \sum_{\beta \leq \mu}\left\|D_{\theta}^{\beta}\left(\theta^{\nu} \hat{\varphi}\right)\right\|=C\left(1-\frac{s}{t}\right) \sum_{\beta \leq \mu}\left\|x^{\beta} D_{x}^{\nu} \varphi\right\| .
$$

Applying (A. 17) we arrive at (A. 26).

\section{References}

[1] Billingsley, P., Convergence of Probability Measures, New York: John Wiley and Sons (1968).

[2] Dawson, D., and Ivanoff, G., Branching Diffusions and Rardom Measures, to appear.

[3] Durrett, R., An infinite particle system with additive interactions, to appear.

[4] Fleischman, J., Limiting distributions for branching random fields, to appear Trans. Amer.Math.Soc.

[5] Ikeda, N., Nagasawa, M., and Watanabe, S., Branching Markov processes I, II, and III, J. Math. Kyoto Univ., 8 (1968), 233-278, 365-410, 9 (1969), 95-110.

[6] Kallenberg, O., Stability of critical cluster fields, to appear.

[7] Martin-Löf, A. Limit theorems for motion of a Pousson system of independent Markovian particles with high density, Z.Wahr. Verw. Geb., 34 (1976) 205-223.

[8] McKean, H. P., Stochastic Integrals, New York: Academic Press (1969).

[9] Meyer, P. A., Le Theoreme de Continuite de P. Levy sur les Espaces Nucleairs. Sem. Bourbaki, 18 e annee, $1965 / 66$ no. 311.

[10] Parthasarathy, K. R., Probability Measures on Metric Space, New York: Academic Press (1969).

[11] Stroock, D. W., Diffusion processes associated with Levy generators, Z. Wahr. Verw. Geb., 32 (1975), 209-244.

[12] Stroock, D. W. and Varadhan, S. R. S., Diffusion processes with boundary conditions, Comm. Pure. Appl. Math., 24 (1971) 147-225.

[13] Dawson, D., Stochastic Evolution Equations and Related Measure Processes, J. Multivariate Anal., 5 (1975), 1-52.

[14] Dawson, D., The Critical Measure Diffusion Process, to appear. 